\title{
Optical surface photometry of a sample of disk galaxies
}

\section{Structural components}

\author{
M. Prieto ${ }^{1}$, J. A. L. Aguerri ${ }^{1,2}$, A. M. Varela ${ }^{1}$, and C. Muñoz-Tuñón ${ }^{1}$ \\ 1 Instituto de Astrofísica de Canarias, 38200 La Laguna, Tenerife, Spain \\ 2 Astronomisches Institut der Universitat Basel, 4102 Binningen, Switzerland
}

Received 13 July 2000 / Accepted 4 December 2000

\begin{abstract}
This work presents the structural decomposition of a sample of 11 disk galaxies, which span a range of different morphological types. The $U, B, V, R$, and $I$ photometric information given in Paper I (color and color-index images and luminosity, ellipticity, and position-angle profiles) has been used to decide what types of components form the galaxies before carrying out the decomposition. We find and model such components as bulges, disks, bars, lenses and rings.
\end{abstract}

Key words. galaxies: spirals - galaxies: structure

\section{Introduction}

The fit of structural models to the luminosity distribution of spiral galaxies is a complicated task. There are several difficulties, among which we emphasize the following:

1) The presence of components other than the bulge and disk. Sometimes these structures are clearly present in the form of, for example, large bars or lenses and we can model them. Often times this is not possible particularly at short wavelengths where the spiral arms, star formation regions and the dust extinction can modify the aspect of the smooth light distribution due to the old population that contributes most of the mass;

2) Bulge models are not unique. All the light-profile functions proposed for ellipticals can be used for the bulges of spirals. In recent years the generalized exponential bulge (Sersic 1968; Sparks 1988) has been the most extensively used model and this law is intrinsically variable;

3) Although exponential disks are well established, it is necessary to have photometric data at large radii in order to avoid contamination from other more central components (Prieto et al. 1992b). Most of the images of nearby galaxies do not reach the low brightness levels to achieve this.

In general, the $B / D$ relation has very little reliability due to these above-mentioned reasons and also because the bulge scale length is sometimes smaller than the seeing disk. Perhaps the most reliable decompositions are those

Send offprint requests to: M. Prieto, e-mail: mpm@ll.iac.es for galaxies with a very well defined disk. This means, well sampled and free from other emission features.

In recent decades much work has been done in order to improve the methods for ascertaining the principal (bulge and disk) components of disk galaxies from their optical photometric profiles. Kormendy (1977); Boroson's (1981); Shombert \& Bothum (1987); Kent (1987); Capaccioli et al. (1987); Andredakis \& Sanders (1994), de Jong (1996); Moriondo et al. (1997). All these studies have resulted in gradual progress in this difficult task.

The majority of the above-mentioned studies analyze the bulge and disk without considering the presence of other components. In this article we try to provide a further step in the unraveling of the complicated structures of spiral galaxies, by taking into account all other components which may be present in disk galaxies. This is done by using the multi-color photometric information (Aguerri et al. (1999), here after Paper I), to determine what the different components are that comprise each galaxy before the decomposition is attempted. We do this for a limited sample of 11 galaxies. In Paper I, we presented the observations and data reduction for this sample of galaxies.

\section{Model fitting}

We use azimuthally averaged profiles to determine the various components. This preserves information on nonaxisymmetric features, such as bars $(m=2)$, because isophotal ellipse fitting allows the ellipticity and the position angle of the $m=2$ structures to be fitted, as reflected in the ellipticity and position angle profiles (Wozniak et al. 1995; Prieto et al. 1997; Varela et al. 1996). However when 
the bar is very large and strong, we characterize the bulge and disk not by azimuthally averaging but by individual profiles transverse to the projected bar.

The profiles are built from ellipses of variable ellipticity and position angle. To consider this variation is a way to correct for warps or any large-scale disk or bulge deformation. The small variations in the luminosity of the disk are thus averaged and the $\mathrm{S} / \mathrm{N}$ increased considerably. Points in the profile affected by additional structures, like rings or tight spiral arms, can easily be omitted.

Moriondo et al. (1997) compared the 1D and 2D techniques and concluded that the $1 \mathrm{D}$ technique is always less accurate and sometimes inconsistent with $2 \mathrm{D}$ one and that the inferior quality of the 1-D fits is attested both by the larger errors in the parameters and by the larger values of $\chi^{2}$. They obtained profiles by keeping the position angle of the ellipses fixed, which introduces an error in the luminosity profile since isophotes are frequently twisted. Moreover, their 2D model considers the apparent bulge and disk ellipticity to be fixed, while in the 1D model, both parameters were allowed to vary. We think that the results of these two analyses are not comparable.

De Jong (1996) also compares the parameters of the bulge and disk fitted in the $1 \mathrm{D}$ and $2 \mathrm{D}$ decompositions in his Fig. 6. The only model used for all the galaxies in their $1 \mathrm{D}$ fit is that of bulge+disk neglecting the obvious presence of bars in many of the galaxies of his sample; however, the bars are taken into account in his $2 \mathrm{D}$ fit. Again, these two analyses cannot be compared.

\subsection{The components}

We assume that the luminosity distribution of a galaxy is the sum of the distributions of its individual components. We use the following laws for fitting the different components found in the galaxy sample:

Bulges: We approximate the bulge luminosity profile to the $r^{1 / n}$ law introduced by Sersic (1968):

$I_{\text {bulge }}(r)=I_{\mathrm{e}} 10^{-b_{n}\left(\left(\frac{r}{r_{\mathrm{e}}}\right)^{1 / n}-1\right)}$,

where $I_{\text {bulge }}(r)$ is the surface brightness of the bulge in flux density at a distance $r$ from the center, or, in surface magnitude units:

$\mu_{\mathrm{b}}(r)=\mu_{\mathrm{e}}+c_{n}\left(\left(\frac{r}{r_{\mathrm{e}}}\right)^{1 / n}-1\right)$,

with $c_{n}=2.5 b_{n}$, and where $b_{n}$ can be chosen in such a way that the scale-radius, $r_{\mathrm{e}}$, is the radius encircling half of the total luminosity, $L_{\mathrm{T}}$, and $I_{\mathrm{e}}$ is the surface brightness at this radius.

Disks: The disk luminosity profiles were fitted by an exponential law, where the surface brightness for the disk in flux density, $I_{\text {disk }}(r)$, is given by:

$I_{\text {disk }}(r)=I_{\text {od }} \mathrm{e}^{-r / h}$.

Or, in surface magnitude units, by,

$\mu_{\mathrm{d}}(r)=\mu_{\mathrm{o}}+1.086 \frac{r}{h}$, where $I_{\mathrm{od}}$ is the central surface brightness of the disk and $h$ is the scale length of the disk.

Bars: We find two types of bars.

1. Elliptical bars. These bars have elliptical isophotes and we have used the two-dimensional function given by Freeman (1966) for fitting the luminosity distribution, $I_{\text {bar }}(x, y)$, of this type of bars:

$I_{\mathrm{bar}}(x, y)=I_{\mathrm{o}, \mathrm{bar}} \sqrt{1-\left(\frac{x}{a_{\mathrm{bar}}}\right)^{2}-\left(\frac{y}{b_{\mathrm{bar}}}\right)^{2}}$

where the free parameters are $I_{\mathrm{o}, \mathrm{bar}}$, the bar central surface brightness, and $a_{\mathrm{bar}}$ and $b_{\mathrm{bar}}$, which are the scale lengths for the semi-major and semi-minor axes, respectively, of the bar. This equation is valid within the bar region (i.e. $x \leq a_{\mathrm{bar}}, y \leq b_{\mathrm{bar}}$ ). In Eq. (5), the origin is the bar center, the $x$-axis is in the direction of the bar major axis and the $y$-axis is perpendicular to this;

2. Flat bars. For fitting this kind of profile we use the expression given in Prieto et al. (1997):

$I_{\mathrm{bar}}(r)=\frac{I_{\mathrm{o}, \mathrm{bar}}}{1+\mathrm{e}^{\frac{r-\alpha}{\beta}}}$

where $\alpha$ and $\beta$ are constants which smooth the end of the bar.

Lenses: A lens is characterized by having a smooth luminosity gradient with a very sharp cut-off. Duval \& Athanassoula (1983), studying the galaxy NGC 5383, found a lens in its luminosity profile and fitted it to the expression:

$I_{\text {lens }}(r)=I_{\mathrm{ol}}\left(1-\left(r / r_{\mathrm{ol}}\right)^{2}\right), r \leq r_{\mathrm{ol}}$

where $I_{\mathrm{ol}}$ is the surface brightness in flux density at the lens center, and $r_{\mathrm{ol}}$ is the typical size of the lens. We used this expression for fitting the lenses found in this sample. Rings: Rings and pseudo-rings are present in many spiral galaxies. The transverse luminosity profiles of these structures are well fitted with Gaussian functions:

$I_{\text {ring }}(r)=I_{\text {or }} \mathrm{e}^{-\frac{1}{2}\left(\frac{r-r_{\text {or }}}{\sigma}\right)^{2}}$,

where $I_{\text {or }}$ is the surface brightness in flux density at $r_{\text {or }}$, which is the radius of the ring, and $\sigma$ is the width of the ring.

The total surface brightness model is the sum of all the above functions corresponding to the components that appear in the galaxy:

$I_{\text {total }}(r)=I_{\text {bulge }}(r)+I_{\text {disk }}(r)+I_{\text {bar }}(r)+I_{\text {lens }}(r)+I_{\text {ring }}+\ldots$

\subsection{The fitting technique}

The most important feature of the method is the detailed study of the multicolor photometric information for each of the galaxies, before performing the decomposition, to determine the different structural components which form 
the galaxies and make an estimate of their scale lengths. After the decomposition we consider whether the parameters obtained are physically meaningful.

For an accurate decomposition, the intrinsic peculiarities of each galaxy do not permit an automatic method. The non-linear $\chi^{2}$ minimization routine method to fit the model profiles to the data points is quicker than interactive methods, and perhaps more suitable when treating a more extensive sample of galaxies, but when we analyze a small number of galaxies in detail, the automatic methods are not useful. For example, some points in the profiles of galaxies with a blue ring must be avoided and the model must pass below them. An automatic method cannot do this. Moreover the $\chi^{2}$ method is not useful for analyzing galaxies with complex structures. The parameters obtained with this method are often not physically meaningful.

The process begins by examining the color and colorindex images, which give us a qualitative idea of what the components are. Next, we examine the color-index profiles, where we can estimate the scale length of any additional components. Then, the confirmation of the assumed components is obtained in the ellipticity and positionangle profiles, where the geometry of the different structures projected onto the sky plane are well reflected. At this point we decide the set of structural components that make up the galaxy. Then we decompose the azimuthally averaged or individual (for galaxies with strong bars) luminosity profiles of the galaxy to obtain the parameters of their components.

We use an interactive profile-fitting routine. The routine begins by fitting the parameters for the disk and bulge over different ranges of the profile, defined by the linear trends of the surface brightness against $r$ and $r^{1 / n}$, respectively, with the least-squares method in an iterative process. Beginning with the estimated initial values, we fit the other components (bars or lenses), by varying their parameters until a good fit is obtained. These were then subtracted from the original profile and the bulge and disk fitted again. This process is repeated until all parameters convergence. We define convergence to have occurred when the difference between the structural parameters of the bulge and disk for two consecutive steps is smaller than the fit errors of those parameters.

For the galaxies, NGC 1300, NGC 7479, and NGC 7723, which have prominent bars, we used profiles along the major and minor axes of the bar instead of the azimuthal profile. On the minor-axis profile (which is less affected by the bar component), corrected for inclination and position angle, we fit the bulge, disk, and other components using the method described above. The fitted bulge and disk functions are subtracted from the major-axis profile to characterize the bar in its long dimension. This process is repeated until all parameters converge as described above. Some bars, such as NGC 7723, and NGC 1300, show very strong star formation regions at their ends, which were fitted with Gaussians. These regions are associated with the beginning of spiral arms. The features created in a profile due to spiral arm crossing it are also well fitted by Gaussian functions.

\section{Structural decomposition of the galaxies}

All the photometric information needed to analyze the galaxies is given in Paper I. The figures in that paper should be viewed in conjunction with this section.

The calibration constants in Paper I include the correction due to galactic inclination and absorption; consequently all parameters are corrected for these effects. We have not corrected for internal extinction. According to Xilouris et al. (1999), a typical face-on spiral galaxy is transparent and is optically thick, at least in the central regions, down to inclination angles of almost $60^{\circ}$. The galaxies in this sample are near to or below these limits.

In Figs. 1-11 we present the decomposition of the luminosity profiles in each filter and in Tables 1-11 we show the parameters of the models as defined in Sect. 2.1, the fractional luminosity of each component, and the ellipticity and position angle of the disk and bar defined as the average value of the last points for the disk and the value at the typical length for the bar.

The uncertainty given for the bulge and disk parameters comes from the "standard errors" of the coefficients of the lines of regression of the fits. These "standard errors" are a measure of the residuals between the observations and the fitted regression line. Clearly, these residuals depend strongly on the deformation of the profile due to structures which were not fitted, such as spiral arms, star formations regions, etc.

The fit for bar and lens parameters was achieved by varying by a fixed amount the parameters of their luminosity laws around the estimated values. This number is the uncertainty in the parameters of the bars and lenses given in Tables $1-11$.

Next, we describe the decomposition procedure for each galaxy. The morphological classification given is that of de Vaucouleurs et al. (1991).

NGC 1300. This is an SB(rs)bc galaxy. In the color and color-index images and profiles (Figs. 1a and 2a, Paper I), we can distinguish a prominent bar of about $70^{\prime \prime}$ (on the major axis), a very blue region at the center, two dust lanes along the bar, curved around the center, which could be related to the presence of an ILR (Athanassoula 1992a,b). Two prominent blue spiral arms, and a uniform color region inside $50^{\prime \prime}$ which suggests the presence of a lens. The bar region has the same color as the disk, suggesting that the stars of the bar are of the same type as those in the surroundings. The galaxy seems to have the following components: a bulge, a disk, a bar, and a lens. The ellipticity profile (Fig. 2a, Paper I) confirms the presence of these components. In Fig. 1 we present the profiles perpendicular and parallel to the bar with the various components fitted. The bulge is fitted with an index $n=4$ in all filters, but not inside $10^{\prime \prime}$ in the perpendicular bar profile, probably due to the strong extinction in this region. The bar is very well fitted by an elliptical function. 

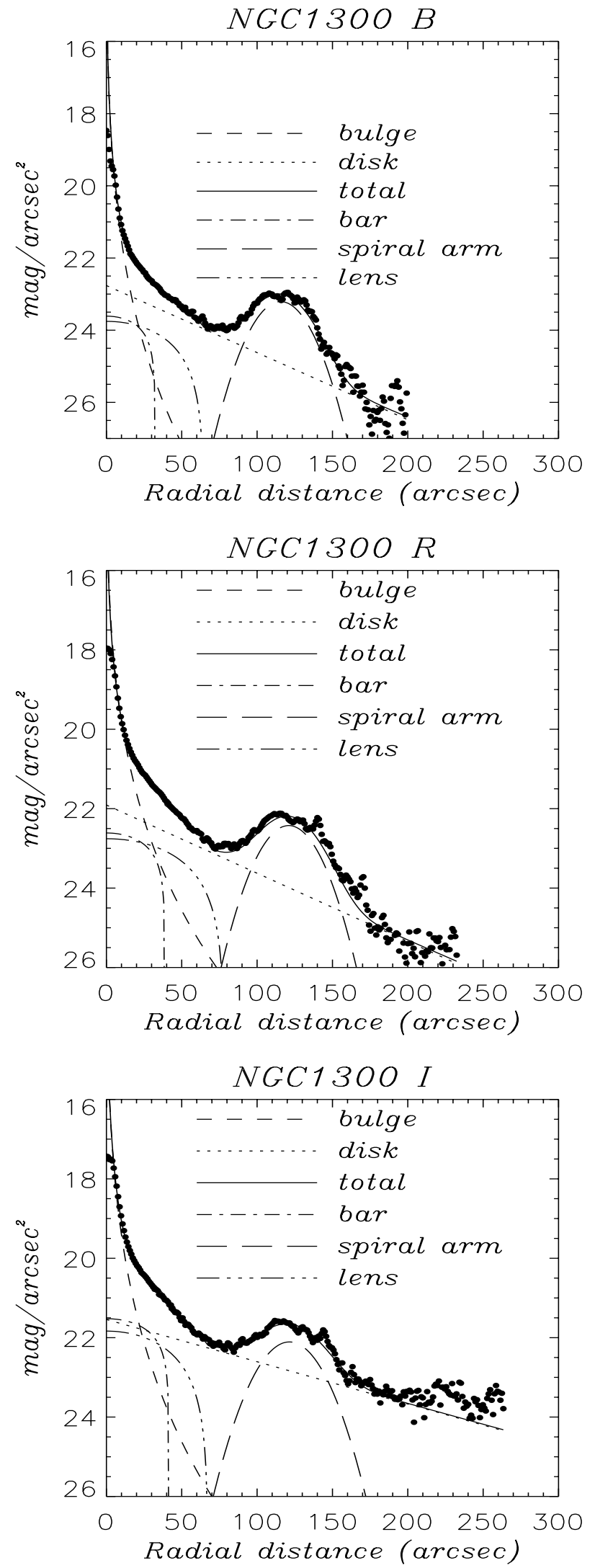
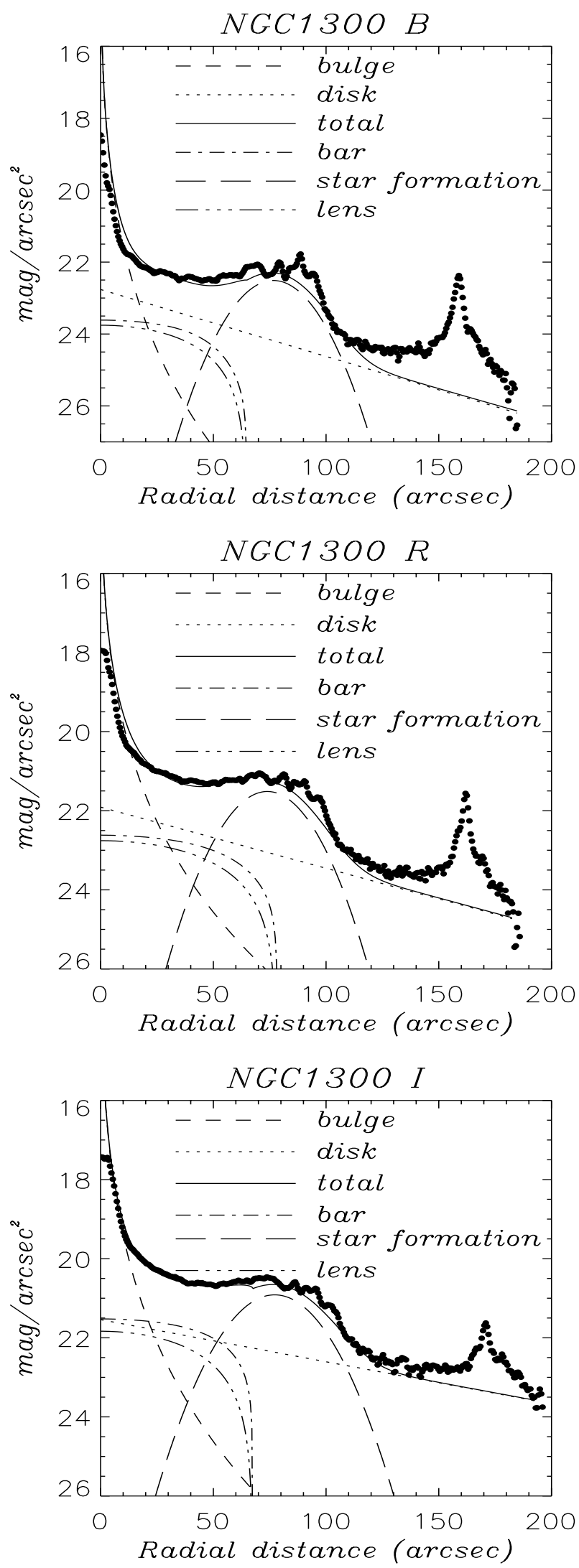

Fig. 1. Structural decomposition of the surface-brightness profiles along the semi-minor bar axis (left) and semi-major bar axis (right) of NGC 1300 in $B, R$, and $I$ 

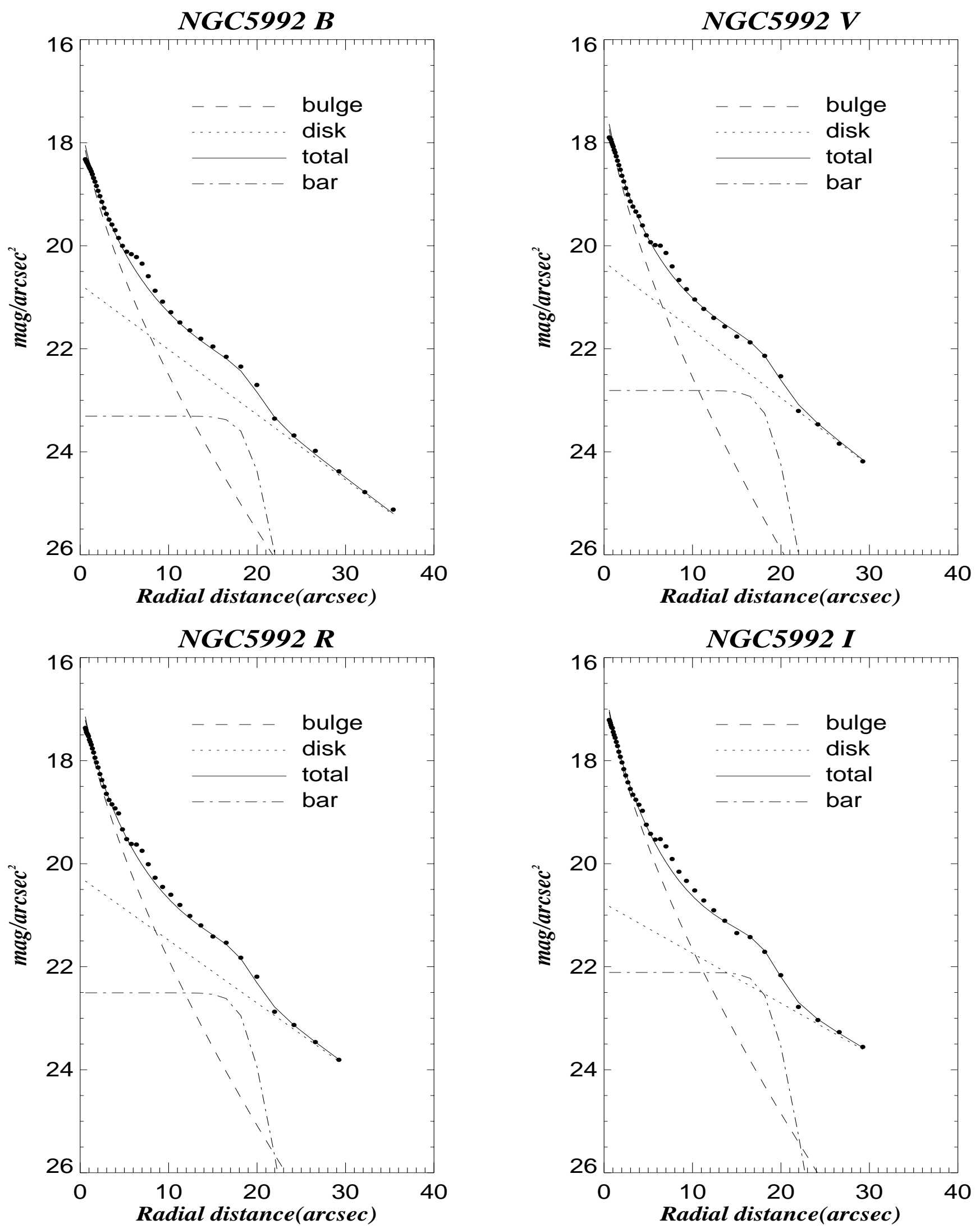

Fig. 2. Structural decomposition of the average surface-brightness profiles of NGC 5992 in $B, V, R$, and $I$ 

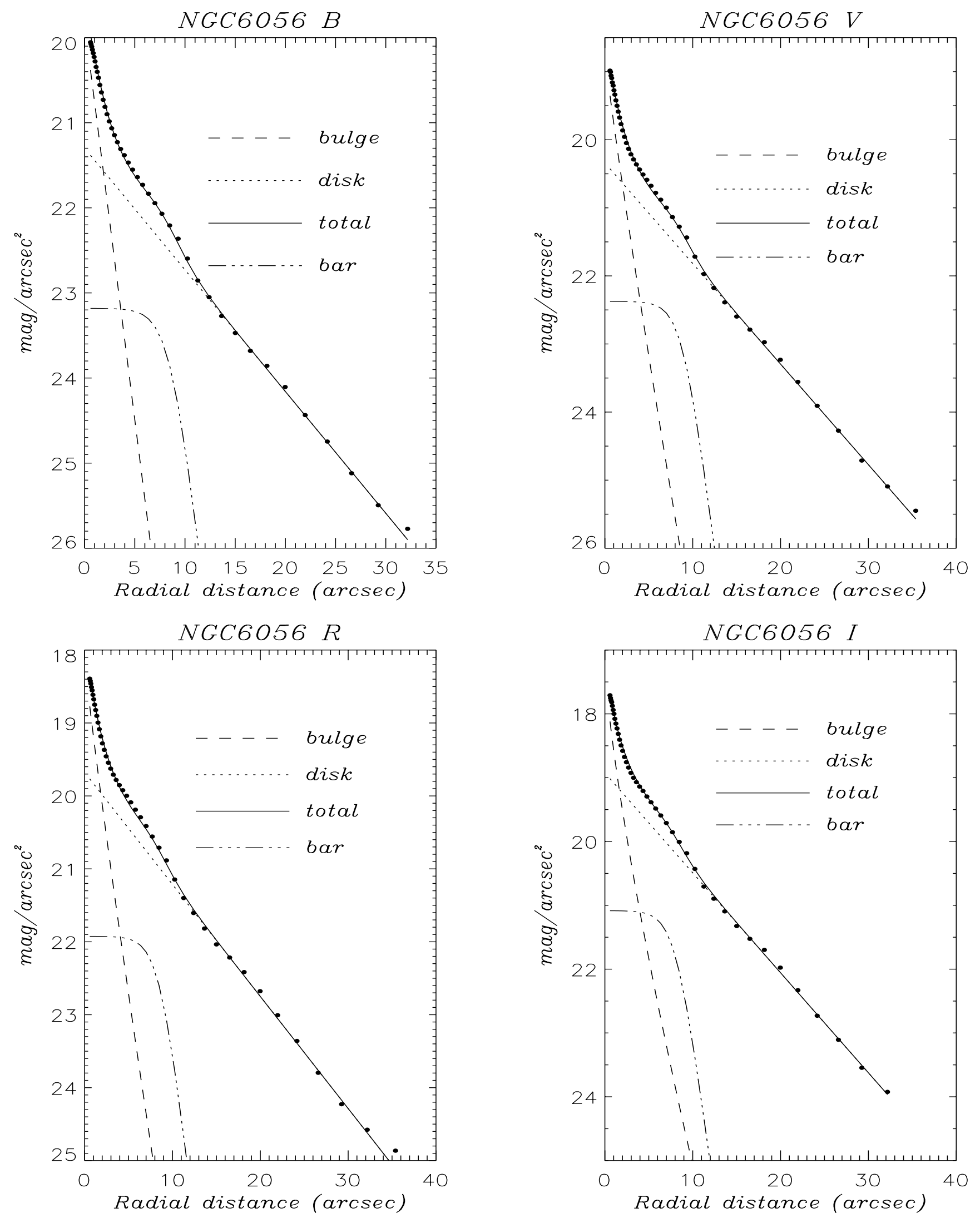

Fig. 3. Structural decomposition of the average surface-brightness profiles of NGC 6056 in $B, V, R$, and $I$ 

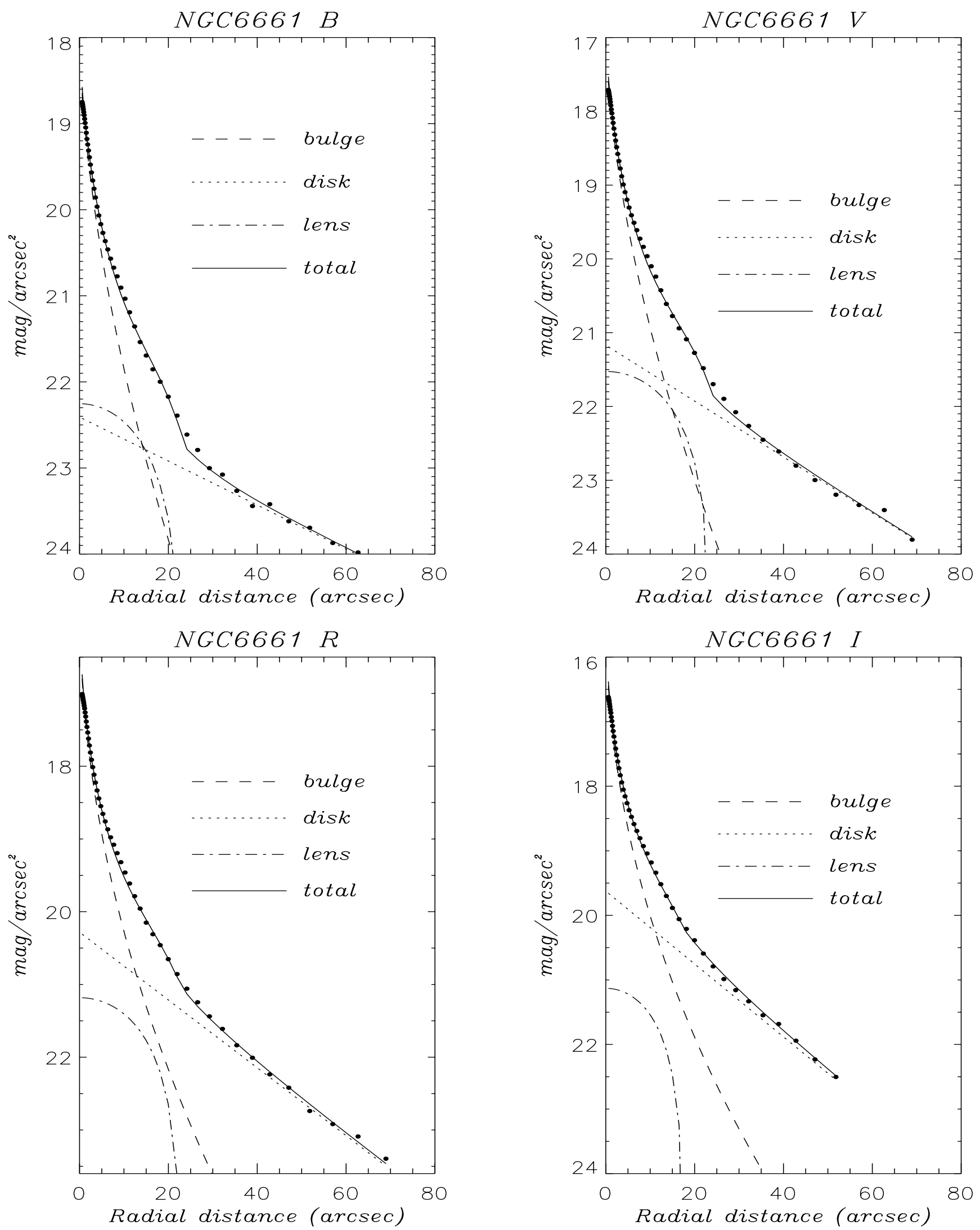

Fig. 4. Structural decomposition of the average surface-brightness profiles of NGC 6661 in $B, V, R$, and $I$ 

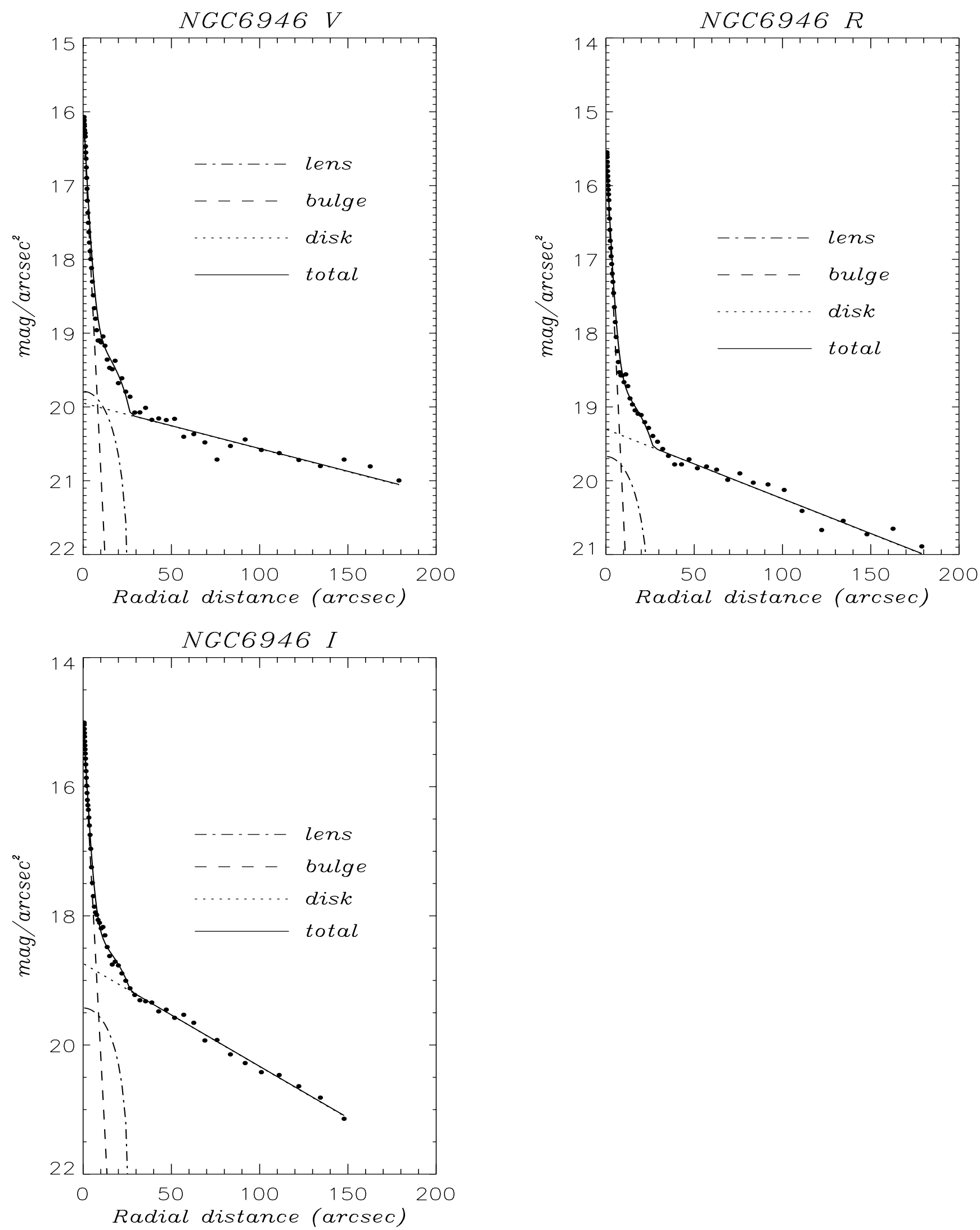

Fig. 5. Structural decomposition of the average surface-brightness profiles of NGC 6946 in $V, R$, and $I$ 

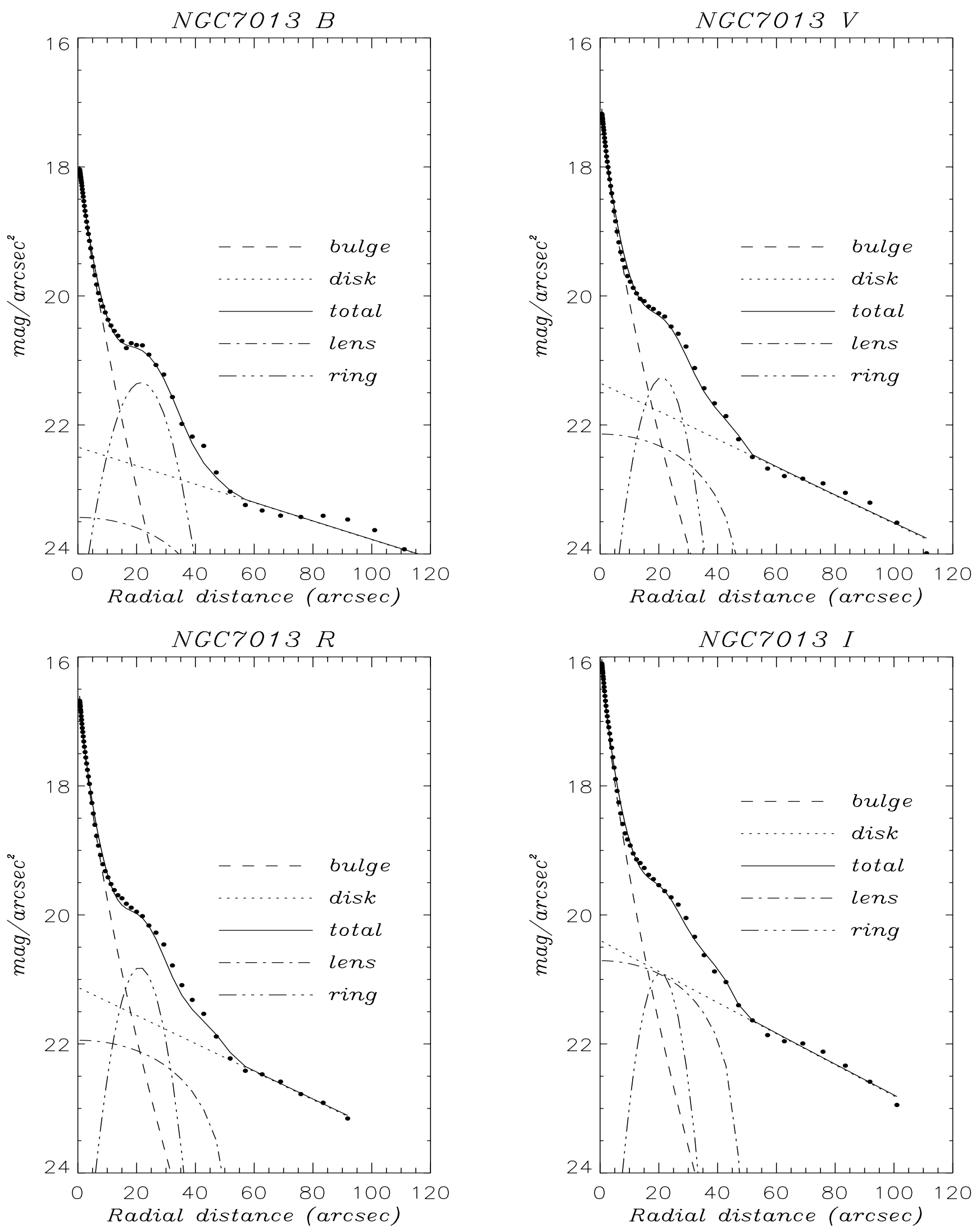

Fig. 6. Structural decomposition of the average surface-brightness profiles of NGC 7013 in $B, V, R$, and $I$ 

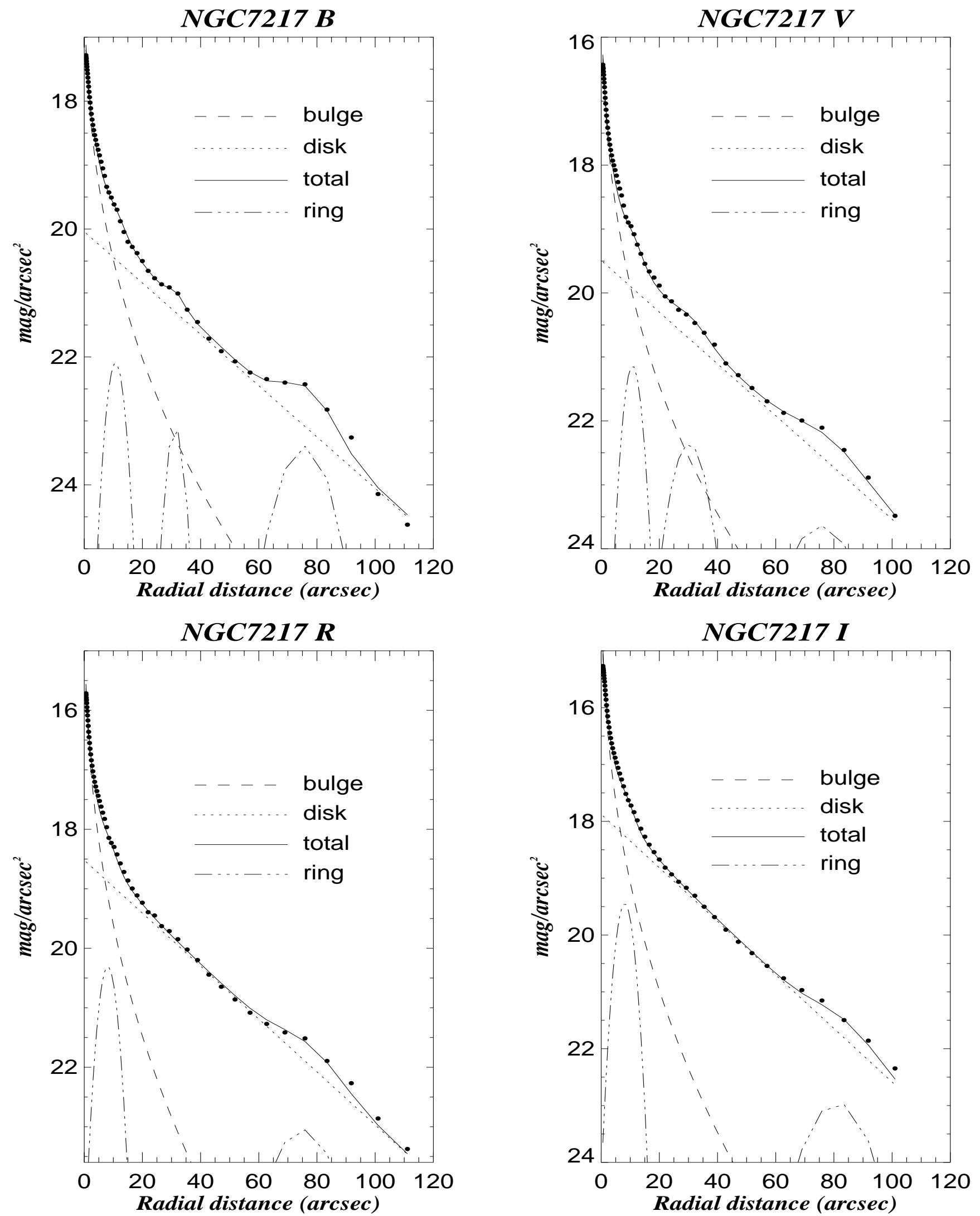

Fig. 7. Structural decomposition of the average surface-brightness profiles of NGC 7217 in $B, V, R$, and $I$ 

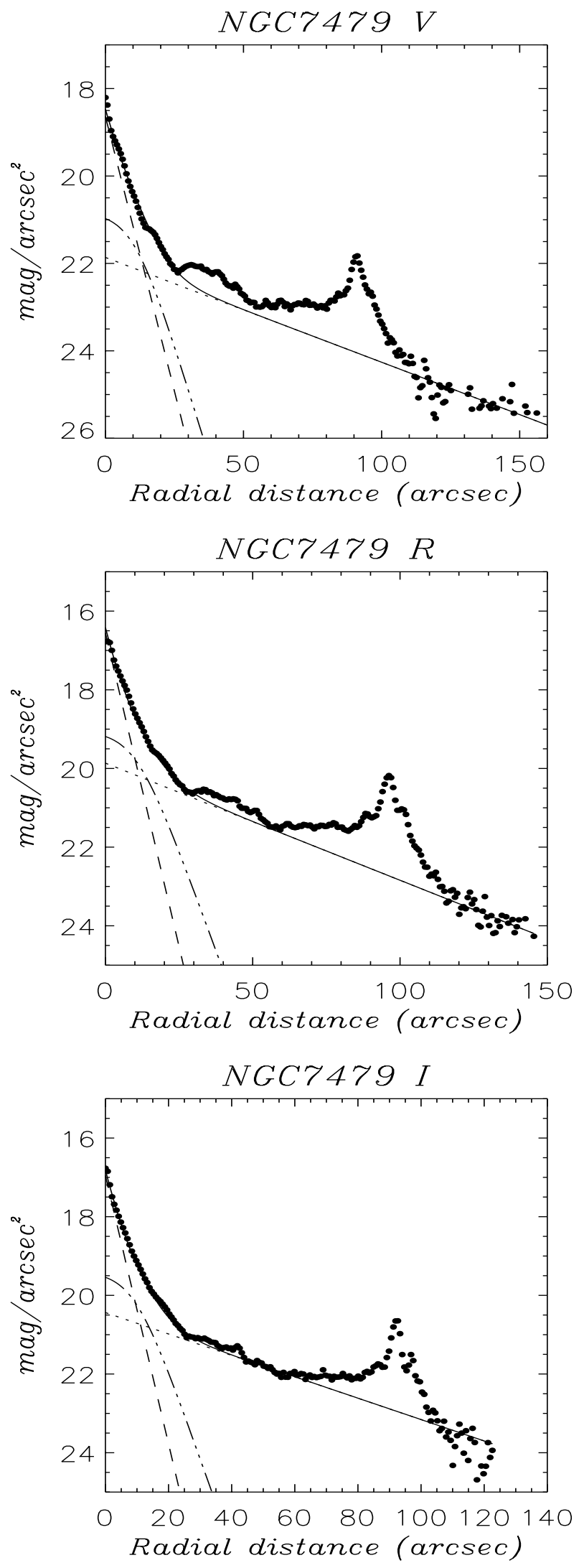
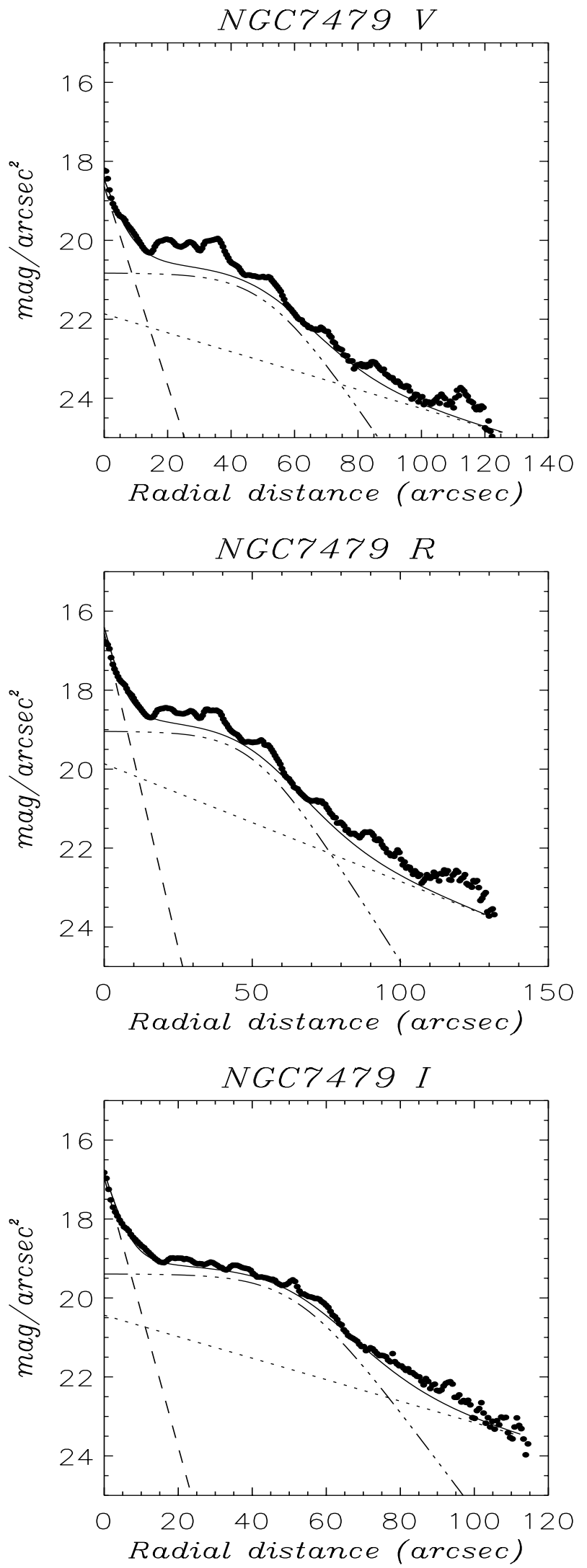

Fig. 8. Structural decomposition of the surface-brightness profiles along the semi-minor bar axis (left) and semi-major bar axis (right) of NGC 7479 in $V, R$, and $I$ 

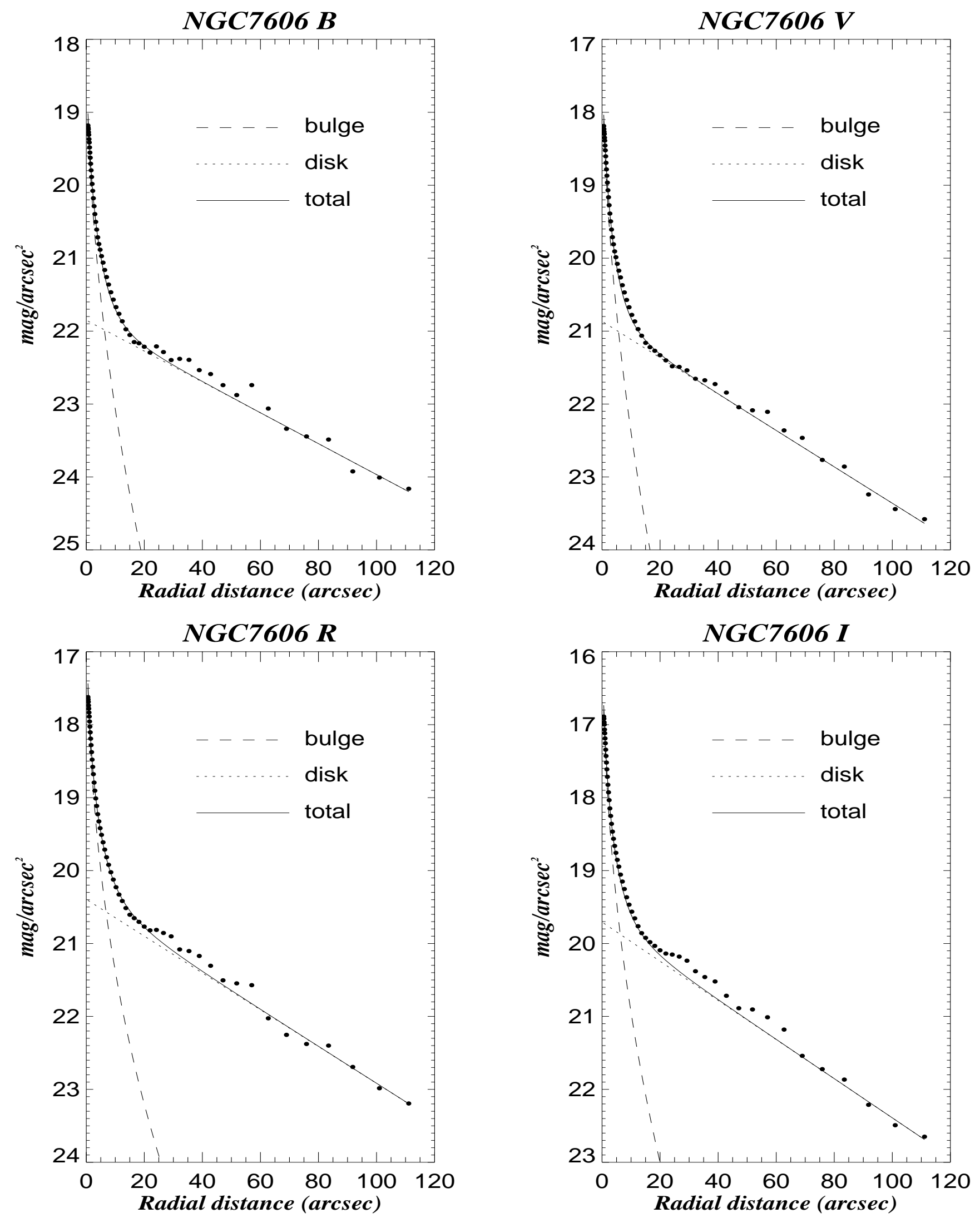

Fig. 9. Structural decomposition of the average surface-brightness profiles of NGC 7606 in $B, V, R$, and $I$ 

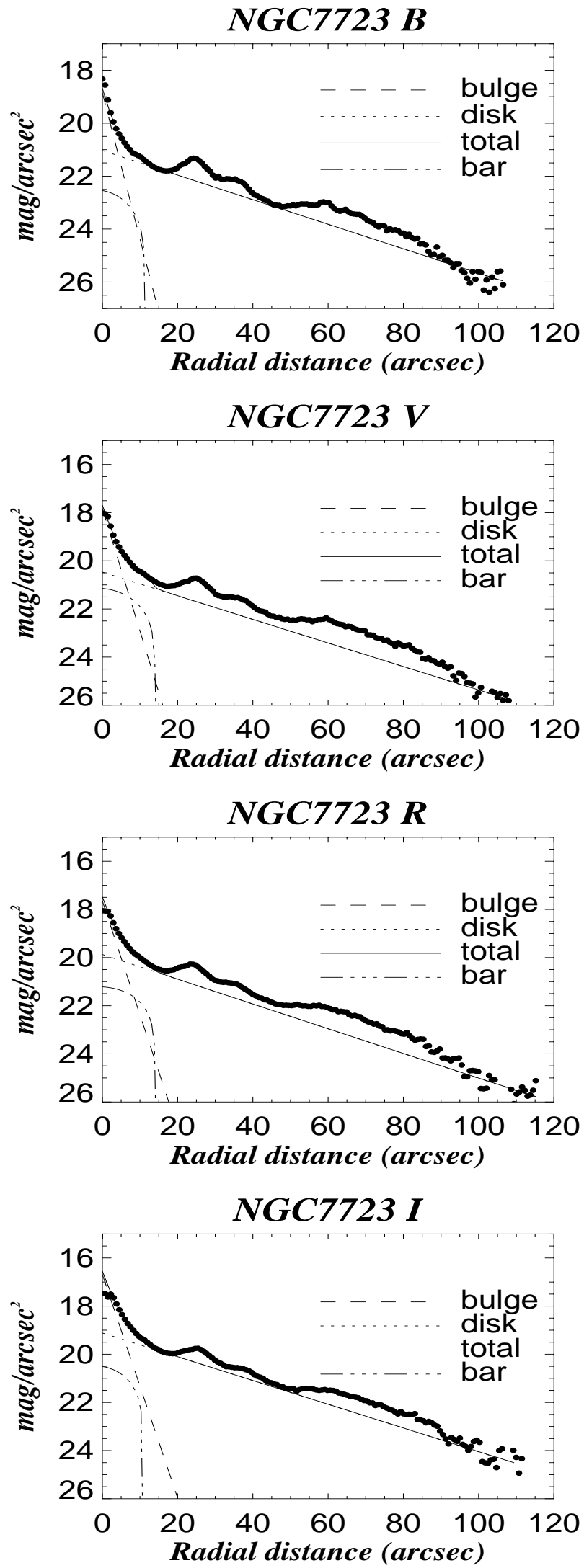
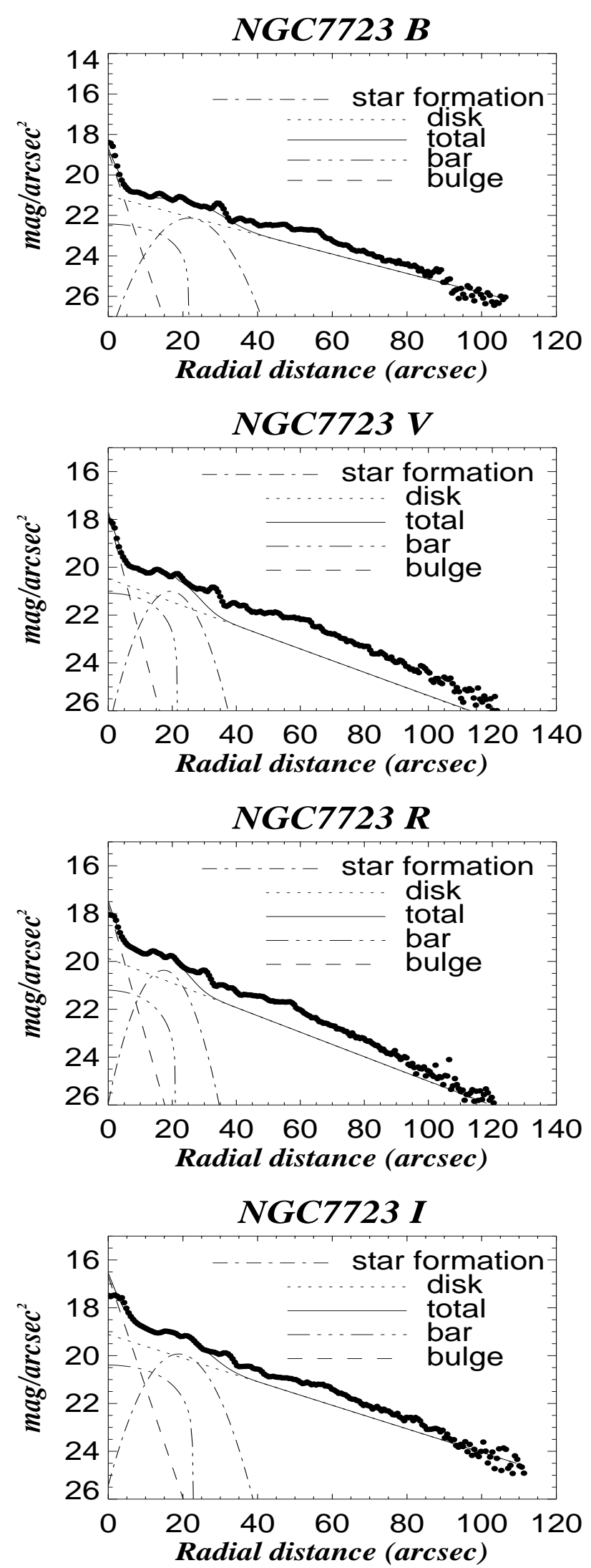

Fig. 10. Structural decomposition of the surface-brightness profiles along the semi-minor bar axis (left) and semi-major bar axis (right) of NGC 7723 in $B, V, R$, and $I$ 

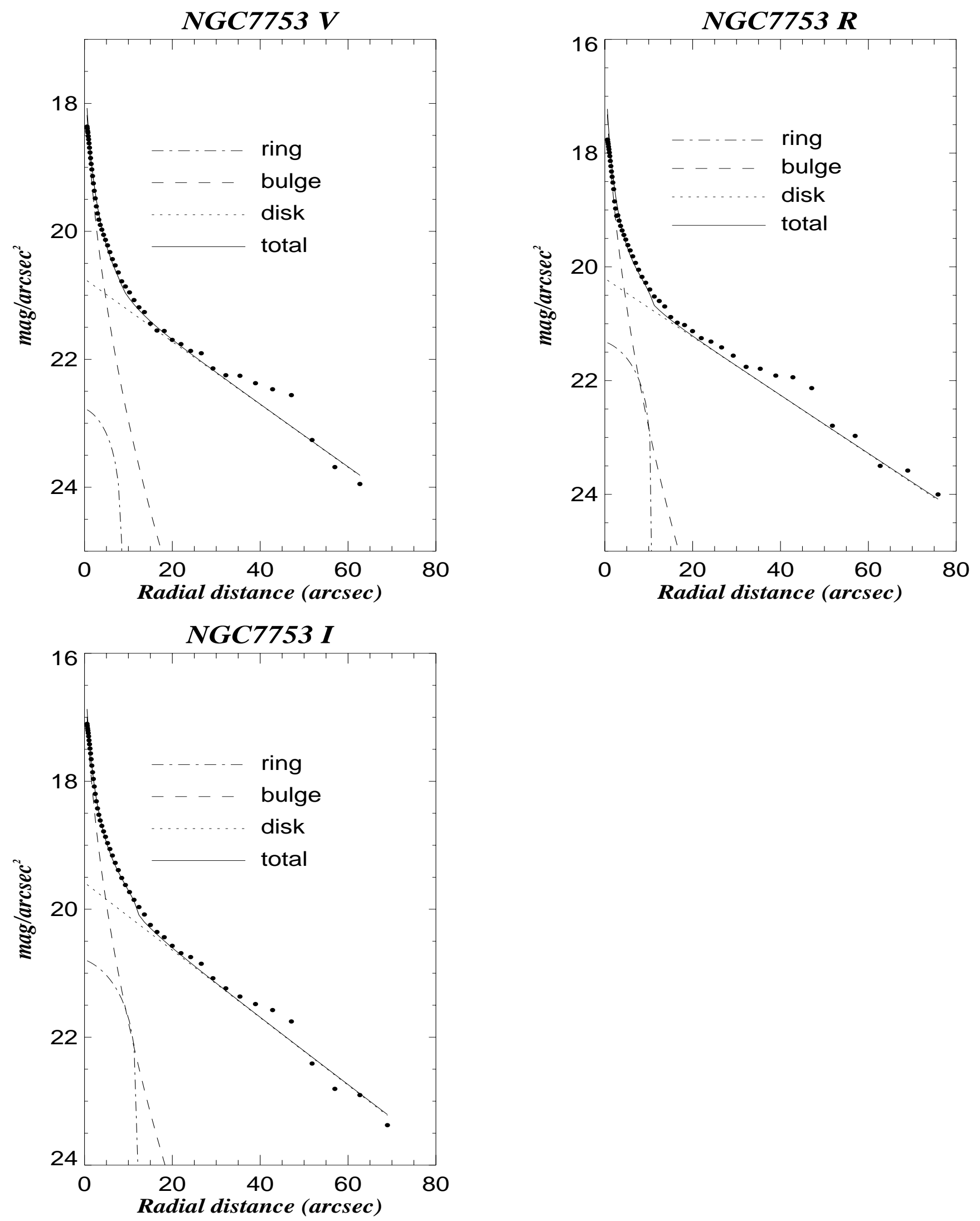

Fig. 11. Structural decomposition of the average surface-brightness profiles of NGC 7753 in $V, R$, and $I$ 
The model for the parallel profiles inside $30^{\prime \prime}$ does not fit the observations well, due to the two dust-lanes parallel to the bar. At the end of the bar there are star formation regions, which are well fitted by a Gaussian function. However, in the parallel profile at $150^{\prime \prime}$ there is a hump in the luminosity due to a spiral arm which is not well fitted by a Gaussian, due the presence in this direction of a very narrow peak, probably caused by a giant star formation region. The hump in the perpendicular profiles around $120^{\prime \prime}$ is a spiral arm which is well fitted by a Gaussian profile. In this galaxy, the errors of the scale lengths of the disk are so large that no conclusions can be drawn concerning the relative values of the parameters of the different components or the bulge-to-disk $(B / D)$ ratio.

NGC 5992. The morphological type of this galaxy is not clear; it is classified as simply S-type by de Vaucouleurs et al. (1991) and as Markarian 489 by Mazzarella \& Balzano (1986). It presents an active starburst nucleus (Balzano 1983; Bicay et al. 1995). In the $B-I$ color-index image (Fig. 1b, Paper I) some structure appears inside $10^{\prime \prime}$, a red region to the NE from the center, and a blue one to the E. In the ellipticity profile (Fig. 2b, Paper I) we observe a feature indicating a bar with a length of about $20^{\prime \prime}$. We fit a luminosity profile for the bulge with an index of 1.5 in all filters, an exponential disk, and a flat bar. The observational feature appearing above the model at about $6.5^{\prime \prime}$ is probably due to the structure within $10^{\prime \prime}$. The $B / D$ ratio is high, which suggests that it is an early-type spiral with structural deformations probably due to an interaction with NGC 5993. In Fig. 2 we present the fit of the all these parameters to the surface-brightness profiles of the galaxies.

NGC 6056: This is a lenticular barred galaxy, classified as SB(s)0. In the color-index map (Fig. 1c, Paper I) we observe a structure of constant color, which probably corresponds to a lens ending at about $20^{\prime \prime}$. The ellipticity and position-angle profiles reveal the presence of a bar about $8^{\prime \prime}$ in length. The lens is very faint in the optical profiles, but is prominent in the infrared filters, as we will show in a future paper. The bar is flat and has a misalignment of about $10^{\circ}$ with respect to the line of nodes of the disk (see Fig. 2c, Paper I). We obtained a very good fit with a flat bar feature. The $r_{\mathrm{e}}$ of the bulge increases, and the scale length of the disk decreases, with redder filters. The scale length of the bar in $I$ is smaller than in the other filters.

NGC 6661. This is a lenticular galaxy, classified as $\mathrm{SA}(\mathrm{s}) 0 / \mathrm{a}$. The color-index images and profiles (Figs. 1d and 2d, Paper I) present a constant-color region from about $10^{\prime \prime}$ to $30^{\prime \prime}$, which suggests the presence of a lens. A red ring appears at about $30^{\prime \prime}$ in the color-index profiles and $B-I$ image. We need to add an elliptical lens to the bulge and disk functions for a good fit to the luminosity profiles in all filters (Fig. 4). The bulge is fitted with an index of about 2 in all filters, the disk is smaller in redder filters, and the scale length of the lens is smaller in $I$ than in the other filters.
NGC 6946. This is a late-type spiral classified as SAB(rs)cd, with an active nucleus, type HII (Keel 1984; Engelbracht et al. 1996). In the $B$ and $U$ filters it was not possible to fit ellipses to the isophotes due to the presence of strong spiral arms and many high luminosity regions, probably star formation regions. The color and color-index images (Figs. 1e and 2e, Paper I) show an extensive central region of constant color, suggesting the presence of a lens. In the color index profile, we can estimate the length scale of this component, about $70^{\prime \prime}$, and this can be confirmed in the ellipticity profiles where there is a change in the ellipticity at just about this distance. The misalignment between the inner and the outer isophotes seen in the position-angle profiles (Fig. 2e, Paper I) may indicate the presence of a triaxial bulge.

The fluctuations of the observations about the disk model (Fig. 6) are due to the presence of spiral arms; there is a very blue arm at about $20^{\prime \prime}$, which causes the model to fall below the observations in the bluer filters. The bulge fits an $r^{1 / 4}$ law, including in the central part, and its relative luminosity increases toward the redder filters. The scale length and relative luminosity of the disk decreases greatly with the redder filters. This galaxy presents the smallest $B / D$ ratio of the sample.

NGC 7013. This is another lenticular galaxy, classified as $\mathrm{SA}(\mathrm{r}) 0 /$ a. Optical and $\mathrm{H}_{\alpha}$ images (Lynds 1974) show a small bulge, and an inner ring. The $\mathrm{H}$ I distribution is in two rings (Eskridge \& Pogge 1991; Knapp et al. 1984). The larger ring is situated just inside the edge of the optical disk, and the smaller one is associated with the inner stellar ring. In the color-index images and profiles (Figs. $1 \mathrm{~g}$ and $2 \mathrm{~g}$, Paper I) we observe a blue ring feature centered at about $25^{\prime \prime}$, and a constant-color region from $10^{\prime \prime}$ to $60^{\prime \prime}$, suggesting the presence of a lens. In the ellipticity profile we can confirm these components. At about $25^{\prime \prime}$ the isophotes have an ellipticity close to 0.65 corresponding to the position of the ring; this is similar to that of the outer disk. After this, there are a plateau until about $60^{\prime \prime}$, which corresponds to the lens. This lens probably has a non-zero intrinsic ellipticity because it is considerably smaller than that of the outer disk, and its position angles are quite different. Beyond this, the ellipticity and position angle of the ellipses are due to the inclination of the galactic disk. We have modeled the observed luminosity profiles of this galaxy with four components (Fig. 6): a bulge, a disk, a lens, and a ring. The value of the index $n$ for the bulge depends on the filter, and is about 1.5. The scale length for the disk and lens is smaller in $I$ than in the other filters.

NGC 7217. This galaxy is classified as (R)SA(r)ab. It is a LINER (Ho et al. 1993; Hummel et al. 1987). In the $B-I$ and $B-V$ color map and profiles (Figs. $1 \mathrm{~g}$ and $2 \mathrm{~g}$, Paper I) we can see the ring structures, with three nuclear rings (red, blue and another red) at about $8^{\prime \prime}, 10^{\prime \prime}$ and $15^{\prime \prime}$, a blue inner ring, and a blue outer ring at about $30^{\prime \prime}$, and $75^{\prime \prime}$, respectively, measured in the $B-I$ image. The ellipticity and PA profiles indicate that the rings are quite circular, and that their isophotes have similar position angles to those of the disk. Buta et al. (1995) and 
Table 1. Structural parameters of NGC 1300

\begin{tabular}{|c|c|c|c|c|}
\hline Structure & Parameters & $B$ & $R$ & $I$ \\
\hline Bulge & $\begin{array}{c}r_{\mathrm{e}}\left({ }^{\prime \prime}\right) \\
\mu_{\mathrm{e}}\left(\mathrm{mag} / \operatorname{arcsec}^{2}\right) \\
n \\
\% L_{\text {Bulge }} / L_{\text {Total }} \\
\end{array}$ & $\begin{array}{c}2.86 \pm 0.17 \\
18.45 \pm 0.16 \\
4.0 \\
30 \pm 1 \\
\end{array}$ & $\begin{array}{c}5.58 \pm 0.15 \\
18.5 \pm 0.1 \\
4.0 \\
29 \pm 1 \\
\end{array}$ & $\begin{array}{c}3.9 \pm 0.3 \\
17.2 \pm 0.2 \\
4.0 \\
19 \pm 1 \\
\end{array}$ \\
\hline Disk & $\begin{array}{c}h\left(^{\prime \prime}\right) \\
\mu_{0}\left(\operatorname{mag} / \operatorname{arcsec}^{2}\right) \\
\text { Ellipticity } \\
\text { Position Angle } \\
\% L_{\text {Disk }} / L_{\text {Total }} \\
B / D \\
\end{array}$ & $\begin{array}{c}58 \pm 21 \\
23 \pm 0.8 \\
0.39 \pm 0.11 \\
96 \pm 10 \\
52 \pm 30 \\
0.5 \pm 0.1\end{array}$ & $\begin{array}{c}70 \pm 10 \\
21.9 \pm 0.5 \\
0.45 \pm 0.07 \\
97 \pm 7 \\
55 \pm 20 \\
0.5 \pm 0.1 \\
\end{array}$ & $\begin{array}{c}101 \pm 25 \\
21.5 \pm 0.8 \\
0.42 \pm 0.08 \\
94 \pm 8 \\
68 \pm 29 \\
0.3 \pm 0.1 \\
\end{array}$ \\
\hline Bar & $\begin{array}{c}\text { Major axis (Type) } \\
\mu_{0}\left(\text { mag } / \operatorname{arcsec}^{2}\right) \\
a\left({ }^{\prime \prime}\right) \\
\text { Minor axis (Type) } \\
b\left(^{\prime \prime}\right) \\
\text { Position angle } \\
\text { Ellipticity }(b / a) \\
\% L_{\text {Bar }} / L_{\text {Total }}\end{array}$ & $\begin{array}{c}\text { Elliptical } \\
23.61 \pm 0.07 \\
95 \pm 1 \\
\text { Elliptical } \\
32.4 \pm 0.5 \\
100 \pm 4 \\
0.34 \pm 0.01 \\
9 \pm 3\end{array}$ & $\begin{array}{c}\text { Elliptical } \\
22.61 \pm 0.03 \\
87 \pm 1 \\
\text { Elliptical } \\
43.2 \pm 0.5 \\
101 \pm 2 \\
0.49 \pm 0.01 \\
7.4 \pm 0.2\end{array}$ & $\begin{array}{c}\text { Elliptical } \\
21.52 \pm 0.02 \\
85 \pm 1 \\
\text { Elliptical } \\
416 \pm 0.5 \\
101 \pm 2 \\
0.43 \pm 0.01 \\
7.67 \pm 0.05\end{array}$ \\
\hline Lens & $\begin{array}{c}\mu_{10}\left(\mathrm{mag} / \operatorname{arcsec}^{2}\right) \\
r_{\mathrm{ol}} \\
\text { Position angle } \\
\% L_{\text {Lens }} / L_{\text {Total }} \\
\end{array}$ & $\begin{array}{c}23.75 \pm 0.07 \\
64.8 \pm 0.5 \\
100 \pm 4 \\
8.15 \pm 0.03\end{array}$ & $\begin{array}{c}22.75 \pm 0.03 \\
78.3 \pm 0.5 \\
100 \pm 4 \\
7.97 \pm 0.14\end{array}$ & $\begin{array}{c}21.83 \pm 0.03 \\
67.5 \pm 0.5 \\
100 \pm 3 \\
5.58 \pm 0.05\end{array}$ \\
\hline
\end{tabular}

Table 2. Structural parameters of NGC 5992

\begin{tabular}{cccccc}
\hline Structure & Parameters & $B$ & $V$ & $R$ & $I$ \\
\hline Bulge & $r_{\mathrm{e}}\left(^{\prime \prime}\right)$ & $4.24 \pm 0.06$ & $3.65 \pm 0.08$ & $3.85 \pm 0.05$ & $3.91 \pm 0.09$ \\
& $\mu_{\mathrm{e}}\left(\mathrm{mag} / \mathrm{arsec}^{2}\right)$ & $20.27 \pm 0.02$ & $19.78 \pm 0.03$ & $19.28 \pm 0.02$ & $19.14 \pm 0.02$ \\
& $n$ & 1.5 & 1.5 & 1.5 & 1.5 \\
& $\% L_{\text {Bulge }} / L_{\text {Total }}$ & $41.2 \pm 0.6$ & $36 \pm 5$ & $45.6 \pm 0.6$ & $47 \pm 2$ \\
\hline Disk & $h\left(^{\prime \prime}\right)$ & $8.63 \pm 0.27$ & $8.18 \pm 0.14$ & $8.85 \pm 0.25$ & $11.15 \pm 0.04$ \\
& $\mu_{0}\left(\mathrm{mag} / \operatorname{arsec}^{2}\right)$ & $20.75 \pm 0.27$ & $20.3 \pm 0.1$ & $20.26 \pm 0.07$ & $20.76 \pm 0.04$ \\
& Ellipticity & $0.22 \pm 0.12$ & $0.23 \pm 0.15$ & $0.21 \pm 0.12$ & $0.21 \pm 0.15$ \\
& Position Angle & $26 \pm 18$ & $26 \pm 23$ & $39 \pm 18$ & $36 \pm 35$ \\
& $\% L_{\text {Disk }} / L_{\text {Total }}$ & $47.98 \pm 0.34$ & $51 \pm 4$ & $42.8 \pm 0.6$ & $38 \pm 2$ \\
& $B / D$ & $0.85 \pm 0.05$ & $0.75 \pm 0.06$ & $1.06 \pm 0.01$ & $1.25 \pm 0.07$ \\
\hline Bar & Type & Flat & Flat & Flat & Flat \\
& $\mu_{0}\left(\right.$ mag $/$ arsec $\left.^{2}\right)$ & $23.3 \pm 0.1$ & $22.8 \pm 0.1$ & $22.5 \pm 0.1$ & $22.1 \pm 0.1$ \\
& $\alpha\left(^{\prime \prime}\right)$ & $19.44 \pm 0.5$ & $18.9 \pm 0.5$ & $18.9 \pm 0.5$ & $18.9 \pm 0.5$ \\
& $\beta\left({ }^{\prime \prime}\right)$ & $1.1 \pm 0.5$ & $1.1 \pm 0.5$ & $1.1 \pm 0.5$ & $1.1 \pm 0.5$ \\
& Position Angle & $89 \pm 3$ & $93 \pm 3$ & $93 \pm 3$ & $93 \pm 3$ \\
& Ellipticity & $0.49 \pm 0.04$ & $0.51 \pm 0.04$ & $0.50 \pm 0.03$ & $0.51 \pm 0.04$ \\
& $\% L_{\text {Bar }} / L_{\text {Total }}$ & $11 \pm 5$ & $13 \pm 6$ & $12 \pm 6$ & $15 \pm 6$ \\
\hline
\end{tabular}

Verdes-Montenegro et al. (1995) studied the ring structures of this galaxy and their locations are in agreement with our values. The bulge is fitted with an $r^{1 / 2}$ law in all filters. In addition to the bulge and disk in the $B$ and $V$ filters, we have fitted the blue nuclear ring, and the blue inner and outer rings; in the $R$ and $I$ filters we have also fitted the red innermost ring and the blue outer ring. All rings are fitted with Gaussian functions. In Table 7, we give the positions of the rings in the different filters from the model fit, as shown in Fig. 7.

NGC 7479. This is a barred galaxy, catalogued as $\mathrm{SB}(\mathrm{s}) \mathrm{bc}$; it is classified as a LINER type by Keel (1983a,b) and Devereux (1989), and as HII (Hummel et al. 1987). This galaxy has been studied by many authors. Dynamic studies by Laine (1996) reveal the presence of an interaction with another galaxy, which could explain the asymmetry of the arms. It presents large, bright H II regions along the bar (Hua et al. 1980). The central blue peak that appears in the $B-I$ color-index profile is probably due to nuclear activity. This galaxy shows a prominent asymmetric bar, and strong spiral arms in the color and color-index images (Figs. 1h and 2h, Paper I). In the bar region there are strong dust lanes, which present an asymmetric distribution. The color of the bar and bulge is redder than 
Table 3. Structural parameters of NGC 6056

\begin{tabular}{cccccc}
\hline Structure & Parameters & $B$ & $V$ & $R$ & $I$ \\
\hline Bulge & $r_{\mathrm{e}}\left(^{\prime \prime}\right)$ & $1.94 \pm 0.02$ & $2.1 \pm 0.2$ & $2.06 \pm 0.07$ & $2.22 \pm 0.13$ \\
& $\mu_{\mathrm{e}}\left(\mathrm{mag} / \mathrm{arcsec}^{2}\right)$ & $21.65 \pm 0.02$ & $20.74 \pm 0.02$ & $20.15 \pm 0.02$ & $19.70 \pm 0.06$ \\
& $n$ & 1.0 & 1.1 & 1.1 & 1.3 \\
& $\% L_{\text {Bulge }} / L_{\text {Total }}$ & $7.54 \pm 0.02$ & $9 \pm 2$ & $9.7 \pm 0.5$ & $9.10 \pm 0.06$ \\
\hline Disk & $h\left(^{\prime \prime}\right)$ & $7.57 \pm 0.07$ & $7.30 \pm 0.12$ & $7.02 \pm 0.05$ & $6.89 \pm 0.15$ \\
& $\mu_{0}\left(\mathrm{mag} / \operatorname{arcsec}^{2}\right)$ & $21.29 \pm 0.02$ & $20.34 \pm 0.06$ & $19.67 \pm 0.07$ & $18.93 \pm 0.08$ \\
& Ellipticity & $0.45 \pm 0.06$ & $0.47 \pm 0.03$ & $0.44 \pm 0.06$ & $0.42 \pm 0.16$ \\
& Position Angle & $53 \pm 5$ & $53 \pm 6$ & $53 \pm 6$ & $54 \pm 15$ \\
& $\% L_{\text {Disk }} / L_{\text {Total }}$ & $83.8 \pm 0.4$ & $83 \pm 3$ & $88 \pm 8$ & $84 \pm 5$ \\
& $B / D$ & $0.10 \pm 0.01$ & $0.11 \pm 0.02$ & $0.11 \pm 0.01$ & $0.11 \pm 0.01$ \\
\hline Bar & Type & Flat & Flat & Flat & Flat \\
& $\mu_{0}($ mag/arcsec & \\
& $\alpha\left(^{\prime \prime}\right)$ & $23.17 \pm 0.03$ & $22.53 \pm 0.02$ & $21.92 \pm 0.02$ & $21.09 \pm 0.02$ \\
& $\beta\left({ }^{\prime \prime}\right)$ & $8.6 \pm 0.5$ & $9.2 \pm 0.5$ & $8.6 \pm 0.5$ & $8.1 \pm 0.5$ \\
& Position Angle & $65 \pm 1$ & $67.7 \pm 0.5$ & $67.5 \pm 0.5$ & $65.4 \pm 0.7$ \\
& Ellipticity & $0.48 \pm 0.01$ & $0.52 \pm 0.01$ & $0.51 \pm 0.01$ & $0.51 \pm 0.01$ \\
& $\% L_{\text {Bar }} / L_{\text {Total }}$ & $8.62 \pm 0.06$ & $8.12 \pm 0.08$ & $7.45 \pm 0.03$ & $7.40 \pm 0.03$ \\
\hline
\end{tabular}

Table 4. Structural parameters of NGC 6661

\begin{tabular}{cccccc}
\hline Structure & Parameters & $B$ & $V$ & $R$ & $I$ \\
\hline Bulge & $r_{\mathrm{e}}\left({ }^{\prime \prime}\right)$ & $7.51 \pm 0.07$ & $7.52 \pm 0.09$ & $7.56 \pm 0.07$ & $7.54 \pm 0.03$ \\
& $\mu_{\mathrm{e}}\left(\mathrm{mag} / \mathrm{arsec}^{2}\right)$ & $21.13 \pm 0.21$ & $20.15 \pm 0.01$ & $19.67 \pm 0.01$ & $19.40 \pm 0.01$ \\
& $n$ & 1.7 & 1.7 & 2.0 & 2.1 \\
& $\% L_{\text {Bulge }} / L_{\text {Total }}$ & $17 \pm 3$ & $27.8 \pm 0.5$ & $35.8 \pm 0.4$ & $32.8 \pm 0.2$ \\
\hline Disk & $\left.h^{\prime \prime}\right)$ & $42 \pm 2$ & $28 \pm 1$ & $28 \pm 1$ & $19 \pm 1$ \\
& $\mu_{0}\left(\mathrm{mag} / \operatorname{arsec}^{2}\right)$ & $21.81 \pm 0.06$ & $21.0 \pm 0.1$ & $21.0 \pm 0.1$ & $19.62 \pm 0.14$ \\
& Ellipticity & $0.46 \pm 0.12$ & $0.34 \pm 0.14$ & $0.30 \pm 0.14$ & $0.3 \pm 0.1$ \\
& Position Angle & $60 \pm 10$ & $56 \pm 14$ & $53 \pm 16$ & $51 \pm 12$ \\
& $\% L_{\text {Disk }} / L_{\text {Total }}$ & $76 \pm 6$ & $63 \pm 1$ & $56 \pm 2$ & $64 \pm 6$ \\
& $B / D$ & $0.22 \pm 0.05$ & $0.44 \pm 0.01$ & $0.64 \pm 0.01$ & $0.51 \pm 0.01$ \\
\hline Lens & $\mu_{10}\left(\mathrm{mag} / \operatorname{arsec}^{2}\right)$ & $21.62 \pm 0.04$ & $21.33 \pm 0.02$ & $21.21 \pm 0.04$ & $21.14 \pm 0.05$ \\
& $r_{\mathrm{ol}}$ & $23.7 \pm 0.5$ & $24.3 \pm 0.5$ & $23.2 \pm 0.5$ & $17.8 \pm 0.5$ \\
& Position Angle & $53 \pm 6$ & $46 \pm 9$ & $49 \pm 3$ & $47 \pm 2$ \\
& $\% L_{\text {Lens }} / L_{\text {Total }}$ & $7.1 \pm 0.1$ & $8.9 \pm 0.1$ & $7.91 \pm 0.03$ & $3.58 \pm 0.01$ \\
\hline
\end{tabular}

that of the disk. The ellipticity and position angle profiles (Fig. 2h, Paper I) have the features of a strong bar about $100^{\prime \prime}$ in semi-length, its position angle being about $100^{\circ}$, different from that of the disk. The presence of a triaxial bulge is suggested by the misalignment between the inner and outer isophotes. The bar is fitted with a flat function along the both axes (Fig. 8). In these profiles, it is possible to see two spiral-arm features, at around $40^{\prime \prime}$ and $100^{\prime \prime}$. This inner spiral arm is very smooth in the $I$ band (it is almost invisible) but is clearly present in the $V$ band. Elmegreen \& Elmegreen (1985), studying the barinterbar intensities, also found that this bar has a flat light profile. The fit is better in the $I$ band than in $R$ and $V$, due the $I$ profile is less affected by the star formation regions present along the bar's major axis (Hummel et al. 1987). The bulge profile fits an exponential function well. As in NGC 1300, the large error in the scale length of the disk prevents us from reaching any conclusions concerning the trend of the parameters with the filters.
NGC 7606. This galaxy is catalogued as $\mathrm{SA}(\mathrm{s}) \mathrm{b}$ with a LINER-type nucleus (Keel 1983b). The color and colorindex images (Figs. 1i and 2i, Paper I) show a red nucleus, a constant-color region inside $20^{\prime \prime}$, and beyond this there are very prominent spiral arms. In the $B-I$ map there are three red pseudo-rings, probably due to the inter regions. The ellipticity and position-angle profiles (Figs. 1i and $2 \mathrm{i}$, Paper I) confirm these structures; inside $20^{\prime \prime}$ the bulge geometry dominates. From here out to about $60^{\prime \prime} \mathrm{a}$ different structure appears, due to the arms and rings, and beyond this distance the ellipticity and position angle are constant due the inclination of the disk. The bulge+disk model provides a good fit to the observations, including the central part of the bulge, with an index $n$ of around 2 for all filters. Andredakis \& Sanders (1994) also found an exponential bulge (Fig. 9). The early type of this galaxy, together with its high inclination, cause the strong spiral arms to look like rings; they are prominent in the isophotal luminosity profiles above the bulge-plus-disk model. 
Table 5. Structural parameters of NGC 6946

\begin{tabular}{ccccc}
\hline Structure & Parameters & $V$ & $R$ & $I$ \\
\hline Bulge & $r_{\mathrm{e}}\left({ }^{\prime \prime}\right)$ & $3.73 \pm 0.01$ & $3.62 \pm 0.02$ & $3.5 \pm 0.1$ \\
& $\mu_{\mathrm{e}}\left(\mathrm{mag} / \operatorname{arcsec}^{2}\right)$ & $17.81 \pm 0.07$ & $17.32 \pm 0.04$ & $16.78 \pm 0.05$ \\
& $\mathrm{n}$ & 1.0 & 1.0 & 1.0 \\
& $\% L_{\text {Bulge }} / L_{\text {Total }}$ & $0.61 \pm 0.03$ & $0.71 \pm 0.12$ & $3 \pm 2$ \\
\hline Disk & $h\left(^{\prime \prime}\right)$ & $174 \pm 21$ & $114 \pm 7$ & $68 \pm 2$ \\
& $\mu_{0}\left(\mathrm{mag} / \operatorname{arcsec}^{2}\right)$ & $19.94 \pm 0.06$ & $19.29 \pm 0.05$ & $18.73 \pm 0.03$ \\
& Ellipticity & $0.67 \pm 0.11$ & $0.63 \pm 0.06$ & $0.58 \pm 0.11$ \\
& Position angle & $101 \pm 7$ & $119 \pm 5$ & $131 \pm 8$ \\
& $\% L_{\text {Disk }} / L_{\text {Total }}$ & $98 \pm 1$ & $98 \pm 1$ & $95 \pm 2$ \\
& $B / D$ & $0.006 \pm 0.002$ & $0.011 \pm 0.001$ & $0.030 \pm 0.001$ \\
\hline Lens & $\mu_{10}\left(\mathrm{mag} / \operatorname{arcsec}^{2}\right)$ & $19.8 \pm 0.1$ & $19.6 \pm 0.1$ & $19.3 \pm 0.1$ \\
& $r_{\text {ol }}$ & $27.5 \pm 0.5$ & $27.5 \pm 0.5$ & $27.5 \pm 0.5$ \\
& Position angle & $110 \pm 4$ & $109 \pm 4$ & $109 \pm 3$ \\
& $\% L_{\text {Lens }} / L_{\text {Total }}$ & $0.67 \pm 0.04$ & $0.64 \pm 0.03$ & $2.2 \pm 0.4$ \\
\hline
\end{tabular}

Table 6. Structural parameters of NGC 7013

\begin{tabular}{cccccc}
\hline Structure & Parameters & $B$ & $V$ & $R$ & $I$ \\
\hline Bulge & $r_{\mathrm{e}}\left({ }^{\prime \prime}\right)$ & $7.37 \pm 0.15$ & $7.56 \pm 0.13$ & $7.74 \pm 0.1$ & $6.41 \pm 0.10$ \\
& $\mu_{\mathrm{e}}\left(\mathrm{mag} / \operatorname{arcsec}^{2}\right)$ & $20.14 \pm 0.02$ & $19.10 \pm 0.03$ & $19.10 \pm 0.01$ & $18.40 \pm 0.04$ \\
& $n$ & 1.3 & 1.5 & 1.6 & 1.5 \\
& $\% L_{\text {Bulge }} / L_{\text {Total }}$ & $12.6 \pm 0.8$ & $25.88 \pm 0.25$ & $23.53 \pm 0.16$ & $19.42 \pm 0.14$ \\
\hline Disk & $h\left(^{\prime \prime}\right)$ & $75 \pm 9$ & $50 \pm 5$ & $55 \pm 4$ & $45 \pm 3$ \\
& $\mu_{0}\left(\mathrm{mag} / \operatorname{arcsec}^{2}\right)$ & $22.3 \pm 0.1$ & $21.3 \pm 0.1$ & $21.3 \pm 0.1$ & $20.40 \pm 0.14$ \\
& Ellipticity & $0.67 \pm 0.05$ & $0.65 \pm 0.08$ & $0.68 \pm 0.05$ & $0.67 \pm 0.11$ \\
& Position Angle & $62 \pm 5$ & $63 \pm 5$ & $64 \pm 4$ & $65 \pm 6$ \\
& $\% L_{\text {Disk }} / L_{\text {Total }}$ & $83 \pm 2$ & $65 \pm 1$ & $66.5 \pm 0.7$ & $66.6 \pm 0.2$ \\
& $B / D$ & $0.15 \pm 0.03$ & $0.29 \pm 0.05$ & $0.34 \pm 0.024$ & $0.3 \pm 0.1$ \\
\hline Ring & $I_{\text {or }}\left(\operatorname{mag} / \operatorname{arcsec}^{2}\right)$ & $21.3 \pm 0.1$ & $21.3 \pm 0.1$ & $20.9 \pm 0.1$ & $20.9 \pm 0.1$ \\
& $r_{\text {or }}\left({ }^{\prime \prime}\right)$ & $21.6 \pm 0.5$ & $21.1 \pm 0.5$ & $20.5 \pm 0.5$ & $20.5 \pm 0.5$ \\
& $\sigma_{\mathrm{r}}\left({ }^{\prime \prime}\right)$ & $8.1 \pm 0.5$ & $6.5 \pm 0.5$ & $6.2 \pm 0.5$ & $5.4 \pm 0.5$ \\
& Ellipticity & $0.48 \pm 0.09$ & $0.44 \pm 0.11$ & $0.56 \pm 0.14$ & $0.5 \pm 0.1$ \\
& Position Angle & $78 \pm 7$ & $80 \pm 8$ & $76 \pm 12$ & $82 \pm 9$ \\
& $\% L_{\text {Ring }} / L_{\text {Total }}$ & $0.83 \pm 0.13$ & $0.52 \pm 0.05$ & $0.72 \pm 0.04$ & $0.33 \pm 0.02$ \\
\hline Lens & $\mu_{l 0}\left(\operatorname{mag} / \operatorname{arcsec}^{2}\right)$ & $23.4 \pm 0.1$ & $22.1 \pm 0.1$ & $21.9 \pm 0.1$ & $20.7 \pm 0.1$ \\
& $r_{\text {ol }}$ & $54 \pm 1$ & $51.3 \pm 0.5$ & $54 \pm 1$ & $48.6 \pm 0.5$ \\
& Position Angle & $71 \pm 3$ & $71 \pm 3$ & $71 \pm 6$ & $74 \pm 2$ \\
& $\% L_{\text {Lens }} / L_{\text {Total }}$ & $3.97 \pm 0.02$ & $8.23 \pm 0.06$ & $9.22 \pm 0.01$ & $14.67 \pm 0.01$ \\
\hline
\end{tabular}

NGC 7723. This is an SB(r)b galaxy with HII-type nuclear activity (Keel 1983b; Hummel et al. 1987; Giurcin et al. 1994). In the color and color-index maps and profiles (Figs. 1j and 2j, Paper I) there is clear evidence for a bar of semi-length about $30^{\prime \prime}$ and a pseudo-ring at the end of the bar. The bar is redder than the disk and shows two straight dust-lanes emanating from the nucleus. The structure of these dust-lanes, which are not curved inward toward the center, suggests that there is no ILR in this galaxy (Athanassoula 1992a,b). The central pixels of this galaxy are saturated and the results are not significant inside the central $5^{\prime \prime}$. The color index of the disk is quite constant with radius. In the ellipticity and position-angle profiles (Fig. 2j, Paper I) the bar and pseudo-ring are clearly present, with different position angles from that of the disk. The bar is well fit by an elliptical bar function, and the pseudo-ring by a Gaussian function. The hump that appears in the perpendicular profiles around $30^{\prime \prime}$ corresponds to the pseudo-ring. This galaxy has smooth and very broad spiral arms, which correspond to the region (from $50^{\prime \prime}$ to $90^{\prime \prime}$ ), where the model does not fit the observed profiles. The bulge is fitted with an index $n=1$ (exponential law). The $r_{\mathrm{e}}$ for the bulge and the $h$ for the disk are constant in all the bands.

NGC 7753. It is in interaction with another galaxy (Salo \& Laurikainen 1993) and is classified as SAB(rs)bc. In the color and color-index maps (Figs. $1 \mathrm{k}$ and $2 \mathrm{k}$, Paper I) a small (about 10" long) bar is evident. The existence of such structure is also indicated by the ellipticity and position-angle profiles (Fig. 2k, Paper I). The luminosity profiles were modeled with a bulge, a disk, and an elliptical bar (Fig. 11). At around 40" there is an increase in luminosity above the model which is due to a spiral arm. The bulge was fitted with an $r^{1 / 2}$ law. 
Table 7. Structural parameters of NGC 7217

\begin{tabular}{|c|c|c|c|c|c|}
\hline Structure & Parameters & $B$ & $\bar{V}$ & $R$ & $I$ \\
\hline Bulge & $\begin{array}{c}r_{\mathrm{e}}\left({ }^{\prime \prime}\right) \\
\mu_{\mathrm{e}}\left(\mathrm{mag} / \operatorname{arcsec}^{2}\right) \\
n \\
\% L_{\text {Bulge }} / L_{\text {Total }} \\
\end{array}$ & $\begin{array}{c}11.21 \pm 0.19 \\
20.70 \pm 0.04 \\
2.5 \\
21.30 \pm 0.23\end{array}$ & $\begin{array}{c}10.72 \pm 0.22 \\
20.03 \pm 0.02 \\
2.8 \\
22.78 \pm 0.58\end{array}$ & $\begin{array}{c}6.96 \pm 0.08 \\
18.82 \pm 0.03 \\
2.5 \\
15.11 \pm 0.05 \\
\end{array}$ & $\begin{array}{c}6.75 \pm 0.10 \\
18.21 \pm 0.02 \\
2.5 \\
15.57 \pm 0.21 \\
\end{array}$ \\
\hline Disk & $\begin{array}{c}h\left({ }^{\prime \prime}\right) \\
\mu_{0}\left(\mathrm{mag} / \operatorname{arcsec}^{2}\right) \\
\text { Ellipticity } \\
\text { Position Angle } \\
\% L_{\text {Disk }} / L_{\text {Total }} \\
B / D \\
\end{array}$ & $\begin{array}{c}26.9 \pm 0.7 \\
20.04 \pm 0.08 \\
0.09 \pm 0.20 \\
91 \pm 8 \\
78.1 \pm 0.9 \\
0.26 \pm 0.01 \\
\end{array}$ & $\begin{array}{c}26.68 \pm 0.17 \\
19.48 \pm 0.03 \\
0.12 \pm 0.05 \\
92 \pm 12 \\
76.6 \pm 0.9 \\
0.29 \pm 0.01 \\
\end{array}$ & $\begin{array}{c}24.26 \pm 0.21 \\
18.51 \pm 0.03 \\
0.12 \pm 0.09 \\
92 \pm 29 \\
84.58 \pm 0.17 \\
0.18 \pm 0.01 \\
\end{array}$ & $\begin{array}{c}22.9 \pm 0.7 \\
17.88 \pm 0.06 \\
0.09 \pm 0.23 \\
92 \pm 57 \\
84.11 \pm 0.48 \\
0.18 \pm 0.01\end{array}$ \\
\hline Blue nuclear ring & $\begin{array}{c}I_{\mathrm{or}}\left(\mathrm{mag} / \operatorname{arcsec}^{2}\right) \\
r_{\mathrm{or}}\left({ }^{\prime \prime}\right) \\
\sigma_{\mathrm{r}}\left({ }^{\prime \prime}\right) \\
\text { Ellipticity } \\
\text { Position Angle } \\
\% L_{\text {Nuclear ring }} / L_{\text {Total }}\end{array}$ & $\begin{aligned} 22.08 & \pm 0.05 \\
10.8 & \pm 0.5 \\
2.7 & \pm 0.5 \\
0.91 & \pm 0.03 \\
73 & \pm 11 \\
0.07 & \pm 0.01\end{aligned}$ & $\begin{array}{c}21.13 \pm 0.02 \\
10.8 \pm 0.5 \\
2.7 \pm 0.5 \\
0.91 \pm 0.02 \\
78 \pm 9 \\
0.1 \pm 0.06\end{array}$ & & \\
\hline Red nuclear ring & 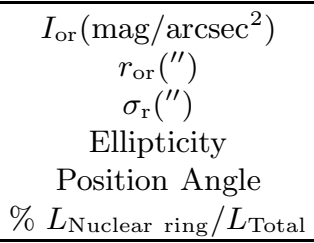 & & & $\begin{array}{c}20.33 \pm 0.05 \\
8.1 \pm 0.5 \\
2 \pm 1 \\
0.97 \pm 0.03 \\
75 \pm 25 \\
0.06 \pm 0.01 \\
\end{array}$ & $\begin{array}{c}19.44 \pm 0.05 \\
8.1 \pm 0.5 \\
2.7 \pm 0.5 \\
0.92 \pm 0.02 \\
74 \pm 7 \\
0.12 \pm 0.02 \\
\end{array}$ \\
\hline Inner ring & $\begin{array}{c}I_{\mathrm{or}}\left(\mathrm{mag} / \operatorname{arcsec}^{2}\right) \\
r_{\mathrm{or}}\left({ }^{\prime \prime}\right) \\
\sigma_{\mathrm{r}}\left({ }^{\prime \prime}\right) \\
\text { Ellipticity } \\
\text { Position Angle } \\
\% L_{\text {Inner ring }} / L_{\text {Total }} \\
\end{array}$ & $\begin{aligned} & 23.1 \pm 0.1 \\
& 31.3 \pm 0.5 \\
& 2.7 \pm 0.5 \\
& 0.83 \pm 0.03 \\
& 83 \pm 7 \\
& 0.08 \pm 0.02 \\
&\end{aligned}$ & $\begin{array}{c}22.35 \pm 0.07 \\
30.2 \pm 0.5 \\
5.4 \pm 0.5 \\
0.86 \pm 0.03 \\
83 \pm 8 \\
0.18 \pm 0.08 \\
\end{array}$ & & \\
\hline Outer ring & $\begin{array}{c}I_{\mathrm{or}}\left(\mathrm{mag} / \operatorname{arcsec}^{2}\right) \\
r_{\mathrm{or}}\left({ }^{\prime \prime}\right) \\
\sigma_{\mathrm{r}}\left({ }^{\prime \prime}\right) \\
\text { Ellipticity } \\
\text { Position Angle } \\
\% L_{\text {Outer ring }} / L_{\text {Total }}\end{array}$ & $\begin{aligned} 23.4 & \pm 0.2 \\
75.6 & \pm 0.5 \\
8.1 & \pm 0.5 \\
0.85 & \pm 0.09 \\
73 & \pm 20 \\
0.44 & \pm 0.03\end{aligned}$ & $\begin{aligned} 23.6 & \pm 0.2 \\
75.6 & \pm 0.5 \\
10.8 & \pm 0.5 \\
0.90 & \pm 0.05 \\
83 & \pm 15 \\
0.28 & \pm 0.05\end{aligned}$ & $\begin{aligned} 23.0 & \pm 0.1 \\
75.1 & \pm 0.5 \\
9.72 & \pm 0.5 \\
0.89 & \pm 0.13 \\
89 & \pm 21 \\
0.24 & \pm 0.06\end{aligned}$ & $\begin{aligned} 22.9 & \pm 0.1 \\
75.6 & \pm 0.5 \\
10.8 & \pm 0.5 \\
0.94 & \pm 0.06 \\
69 & \pm 27 \\
0.18 & \pm 0.09\end{aligned}$ \\
\hline
\end{tabular}

\section{Discussion}

To analyze the results of the structural decomposition of this sample of galaxies, we divide these in three groups: those with a well defined disk, those with an ill-defined disk and those with strong bars and well developed arms.

1.- Galaxies with a well defined disk.

These galaxies have an extensive part of their disk free from contamination by other components, which implies that the fitted bulge and disk parameters are reliable, and that we can make comparisons between them. Our criterion for selecting these galaxies is that they have at least $1 / 3$ of their luminosity profile coming from the disk without overlapping other components. Only four galaxies obey this condition: NGC 6056, NGC 6661, NGC 6946 , and NGC 7606 (NGC 6661 fault this condition in the $I$ band.

The following interpretations, although of an outstandingly coherent behavior, are made with caution due the small number of galaxies and the reduced statistical significance.
In Fig. 12 we present the shape index, $n$, versus the filter. There is a trend where $n$ increases with the redder filters. This can be interpreted as the older population being more extended than the younger, which could indicate an end of the star formation in the bulge from outside to inside. Extinction would have the reverse trend. The absolute variation of $n$ with filter is \pm 0.3 around the mean value. The $n$ index is bigger for the $\mathrm{Sb}$ galaxy than for the Scd one. We are not going to compare this index between spirals and lenticulars.

These galaxies are of different morphological types ( $\mathrm{SO}, \mathrm{SO} / \mathrm{a}, \mathrm{Sb}$, and $\mathrm{Sc}$ ). Their inclinations are the largest of the sample, near the transparent limits $\left(60^{\circ}\right)$ (Xilouris et al. 1999), so we expect to find some effect of the internal extinction on the scale length of the disk, $h$, depending on the filter. In Fig. 13 we see how $h$ varies with the filter for each galaxy. The scale length of the disk decreases in all galaxies when the filter is redder, as we would expect an optical thickness increasing towards the center of the galaxy. However, we cannot exclude an additional effect 
Table 8. Structural parameters of NGC 7479

\begin{tabular}{|c|c|c|c|c|}
\hline Structure & Parameters & $V$ & $R$ & $I$ \\
\hline Bulge & $\begin{array}{c}r_{\mathrm{e}}\left({ }^{\prime \prime}\right) \\
\mu_{\mathrm{e}}\left(\mathrm{mag} / \operatorname{arcsec}^{2}\right) \\
n \\
\% L_{\text {Bulge }} / L_{\text {Total }} \\
\end{array}$ & $\begin{array}{c}7.22 \pm 0.09 \\
20.45 \pm 0.19 \\
1 \\
11.32 \pm 0.18 \\
\end{array}$ & $\begin{array}{c}5.7 \pm 0.4 \\
19.39 \pm 0.04 \\
1 \\
10.98 \pm 0.32 \\
\end{array}$ & $\begin{array}{c}5.3 \pm 0.4 \\
18.77 \pm 0.09 \\
1 \\
9.68 \pm 0.08 \\
\end{array}$ \\
\hline Disk & $\begin{array}{c}h\left(^{\prime \prime}\right) \\
\mu_{0}\left(\mathrm{mag} / \operatorname{arcsec}^{2}\right) \\
\text { Ellipticity } \\
\text { Position Angle } \\
\% L_{\text {Disk }} / L_{\text {Total }} \\
B / D \\
\end{array}$ & $\begin{array}{c}45 \pm 5 \\
21.86 \pm 0.19 \\
0.24 \pm 0.06 \\
118 \pm 9 \\
62.65 \pm 0.15 \\
0.17 \pm 0.02 \\
\end{array}$ & $\begin{array}{c}36 \pm 6 \\
20.9 \pm 0.8 \\
0.26 \pm 0.05 \\
116 \pm 7 \\
57.05 \pm 0.15 \\
0.18 \pm 0.05 \\
\end{array}$ & $\begin{aligned} & 40 \pm 3 \\
& 20.44 \pm 0.14 \\
& 0.25 \pm 0.08 \\
& 119 \pm 11 \\
& 61.93 \pm 0.15 \\
& 0.15 \pm 0.04 \\
&\end{aligned}$ \\
\hline Bar & $\begin{array}{c}\text { Major axis (Type) } \\
\mu_{0}\left(\text { mag } / \operatorname{arcsec}^{2}\right) \\
\alpha\left(^{\prime \prime}\right) \\
\beta\left(\left(^{\prime \prime}\right)\right. \\
\text { Minor axis (Type) } \\
\alpha\left(^{\prime \prime}\right) \\
\beta\left(^{\prime \prime}\right) \\
\text { Position Angle } \\
\text { Ellipticity } \\
\% L_{\text {Bar }} / L_{\text {Total }}\end{array}$ & $\begin{array}{c}\text { Flat } \\
20.83 \pm 0.01 \\
51.0 \pm 0.5 \\
9.2 \pm 0.5 \\
\text { Flat } \\
19.0 \pm 1.0 \\
9.8 \pm 0.5 \\
98 \pm 2 \\
0.76 \pm 0.02 \\
26.03 \pm 0.13\end{array}$ & $\begin{array}{c}\text { Flat } \\
20.03 \pm 0.01 \\
51.0 \pm 0.5 \\
9.2 \pm 0.5 \\
\text { Flat } \\
19.0 \pm 1.0 \\
9.8 \pm 0.5 \\
99 \pm 2 \\
0.74 \pm 0.02 \\
31.95 \pm 0.11\end{array}$ & $\begin{array}{c}\text { Flat } \\
19.39 \pm 0.01 \\
52.0 \pm 0.5 \\
8.6 \pm 0.5 \\
\text { Flat } \\
17.5 \pm 0.5 \\
9.2 \pm 0.5 \\
99 \pm 2 \\
0.72 \pm 0.02 \\
28.38 \pm 0.18\end{array}$ \\
\hline
\end{tabular}

Table 9. Structural parameters of NGC 7606

\begin{tabular}{cccccc}
\hline Structure & Parameters & $B$ & $V$ & $R$ & $I$ \\
\hline Bulge & $r_{\mathrm{e}}\left({ }^{\prime \prime}\right)$ & $5.83 \pm 0.11$ & $4.99 \pm 0.05$ & $7.4 \pm 0.2$ & $5.86 \pm 0.06$ \\
& $\mu_{\mathrm{e}}\left(\mathrm{mag} / \operatorname{arcsec}^{2}\right)$ & $21.84 \pm 0.02$ & $20.73 \pm 0.01$ & $20.74 \pm 0.03$ & $19.74 \pm 0.02$ \\
& $n$ & 2.1 & 2.0 & 2.5 & 2.3 \\
& $\% L_{\text {Bulge }} / L_{\text {Total }}$ & $3 \pm 1$ & $4 \pm 1$ & $5.84 \pm 0.24$ & $6 \pm 1$ \\
\hline Disk & $h\left(^{\prime \prime}\right)$ & $51 \pm 6$ & $43 \pm 5$ & $43 \pm 4$ & $40 \pm 4$ \\
& $\mu_{0}\left(\mathrm{mag} / \operatorname{arcsec}^{2}\right)$ & $21.85 \pm 0.25$ & $20.9 \pm 0.3$ & $20.39 \pm 0.25$ & $19.71 \pm 0.25$ \\
& Ellipticity & $0.59 \pm 0.03$ & $0.59 \pm 0.02$ & $0.59 \pm 0.02$ & $0.59 \pm 0.02$ \\
& Position Angle & $53 \pm 2$ & $56 \pm 2$ & $56 \pm 2$ & $56 \pm 2$ \\
& $\% L_{\text {Disk }} / L_{\text {Total }}$ & $96.8 \pm 0.7$ & $96 \pm 1$ & $94 \pm 2$ & $94.7 \pm 0.7$ \\
& $B / D$ & $0.034 \pm 0.001$ & $0.041 \pm 0.003$ & $0.067 \pm 0.007$ & $0.058 \pm 0.002$ \\
\hline
\end{tabular}

Table 10. Structural parameters of NGC 7723

\begin{tabular}{|c|c|c|c|c|c|}
\hline Structure & Parameters & $B$ & $V$ & $R$ & $I$ \\
\hline Bulge & $\begin{array}{c}r_{\mathrm{e}}\left({ }^{\prime \prime}\right) \\
\mu_{\mathrm{e}}\left(\mathrm{mag} / \operatorname{arcsec}^{2}\right) \\
n \\
\% L_{\text {Bulge }} / L_{\text {Total }} \\
\end{array}$ & $\begin{array}{c}3 \pm 1 \\
20.66 \pm 0.06 \\
1 \\
5 \pm 4 \\
\end{array}$ & $\begin{array}{c}3.55 \pm 0.14 \\
19.63 \pm 0.08 \\
1 \\
9 \pm 1 \\
\end{array}$ & $\begin{array}{c}3.79 \pm 0.04 \\
19.46 \pm 0.04 \\
1 \\
8 \pm 1 \\
\end{array}$ & $\begin{array}{c}3.91 \pm 0.02 \\
18.49 \pm 0.01 \\
1 \\
9.25 \pm 0.05 \\
\end{array}$ \\
\hline Disk & $\begin{array}{c}h\left(^{\prime \prime}\right) \\
\mu_{0}\left(\mathrm{mag} / \operatorname{arcsec}^{2}\right) \\
\text { Ellipticity } \\
\text { Position Angle } \\
\% L_{\text {Disk }} / L_{\text {Total }} \\
B / D \\
\end{array}$ & $\begin{array}{c}23 \pm 7 \\
21 \pm 2 \\
0.31 \pm 0.08 \\
127 \pm 3 \\
91 \pm 14 \\
0.06 \pm 0.35\end{array}$ & $\begin{array}{c}22 \pm 4 \\
21 \pm 1 \\
0.31 \pm 0.06 \\
129 \pm 7 \\
82 \pm 10 \\
0.1 \pm 0.1 \\
\end{array}$ & $\begin{array}{c}21 \pm 3 \\
20 \pm 1 \\
0.32 \pm 0.08 \\
128 \pm 9 \\
86 \pm 8 \\
0.09 \pm 0.07\end{array}$ & $\begin{array}{c}22 \pm 5 \\
19.12 \pm 0.01 \\
0.31 \pm 0.02 \\
126 \pm 2 \\
86.1 \pm 0.7 \\
0.1 \pm 0.1\end{array}$ \\
\hline Bar & $\begin{array}{c}\text { Major axis }(\text { Type }) \\
\mu_{0}\left(\text { mag } / \operatorname{arcsec}^{2}\right) \\
a\left({ }^{\prime \prime}\right) \\
\text { Minor axis (type) } \\
b\left(^{\prime \prime}\right) \\
\text { Position angle } \\
\text { Ellipticity } \\
\% L_{\text {Bar }} / L_{\text {Total }}\end{array}$ & $\begin{array}{c}\text { Elliptical } \\
22.41 \pm 0.02 \\
22 \pm 1 \\
\text { Elliptical } \\
11 \pm 1 \\
159 \pm 4 \\
0.5 \pm 0.1 \\
3.81 \pm 0.32\end{array}$ & $\begin{array}{c}\text { Elliptical } \\
21.15 \pm 0.01 \\
22 \pm 1 \\
\text { Elliptical } \\
13 \pm 1 \\
157 \pm 5 \\
0.59 \pm 0.07 \\
8.87 \pm 0.08\end{array}$ & $\begin{array}{c}\text { Elliptical } \\
21.02 \pm 0.01 \\
21 \pm 1 \\
\text { Elliptical } \\
14 \pm 1 \\
157 \pm 4 \\
0.66 \pm 0.07 \\
6.79 \pm 0.07\end{array}$ & $\begin{array}{c}\text { Elliptical } \\
20.3 \pm 0.01 \\
23 \pm 1 \\
\text { Elliptical } \\
11 \pm 1 \\
156 \pm 3 \\
0.47 \pm 0.06 \\
5.02 \pm 0.04\end{array}$ \\
\hline
\end{tabular}


Table 11. Structural parameters of NGC 7753

\begin{tabular}{ccccc}
\hline Structure & Parameters & $V$ & $R$ & $I$ \\
\hline Bulge & $\left.r_{\mathrm{e}}{ }^{\prime \prime}\right)$ & $4 \pm 1$ & $3 \pm 1$ & $4 \pm 1$ \\
& $n$ & $20.64 \pm 0.12$ & $19.54 \pm 0.23$ & $19.45 \pm 0.09$ \\
& $\mu_{\mathrm{e}}\left(\mathrm{mag} / \operatorname{arcsec}^{2}\right)$ & 2 & 2 & 2 \\
& $\% L_{\text {Bulge }} / L_{\text {Total }}$ & $9 \pm 2$ & $7 \pm 1$ & $12 \pm 2$ \\
\hline Disk & $h\left(^{\prime \prime}\right)$ & $22 \pm 3$ & $21 \pm 4$ & $20 \pm 3$ \\
& $\mu_{0}\left(\mathrm{mag} / \operatorname{arcsec}^{2}\right)$ & $20.64 \pm 0.02$ & $20.18 \pm 0.01$ & $19.64 \pm 0.01$ \\
& Ellipticity & $0.2 \pm 0.1$ & $0.2 \pm 0.1$ & $0.2 \pm 0.1$ \\
& Position angle & $150 \pm 10$ & $147 \pm 11$ & $150 \pm 10$ \\
& $\% L_{\text {Disk }} / L_{\text {Total }}$ & $90 \pm 2$ & $91 \pm 1$ & $85 \pm 3$ \\
& $B / D$ & $0.09 \pm 0.04$ & $0.08 \pm 0.05$ & $0.14 \pm 0.09$ \\
\hline Bar & Type & Elliptical & Elliptical & Elliptical \\
& $\mu_{0}($ mag/arcsec & \\
& $a\left({ }^{\prime \prime}\right)$ & $22.29 \pm 0.01$ & $21.20 \pm 0.03$ & $20.41 \pm 0.05$ \\
& Position angle & $8.6 \pm 0.5$ & $10.8 \pm 0.5$ & $12.4 \pm 0.5$ \\
& Ellipticity & $170 \pm 3$ & $171 \pm 2$ & $175 \pm 3$ \\
& $\% L_{\text {Bar }} / L_{\text {Total }}$ & $0.45 \pm 0.03$ & $0.45 \pm 0.02$ & $0.44 \pm 0.03$ \\
& & $0.88 \pm 0.08$ & $2.24 \pm 0.12$ & $4.31 \pm 0.23$ \\
\hline
\end{tabular}

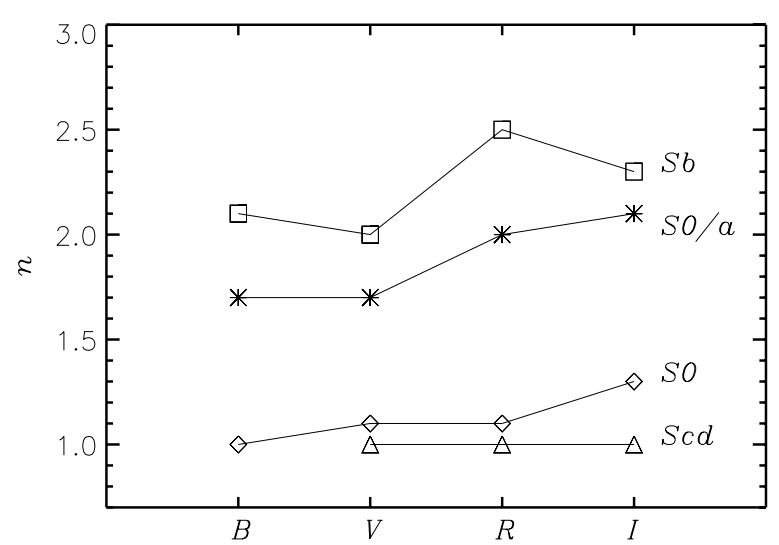

Fig. 12. The index $n$ of the bulge model vs. filter for those galaxies with well defined disk and variable index
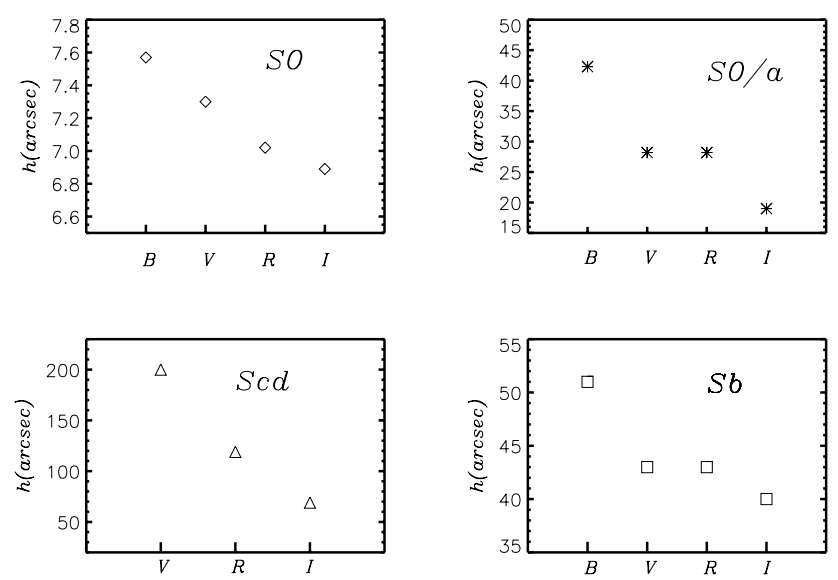

Fig. 13. Scale length of the disk against filter for those galaxies with well defined disk

due to stellar population. The effective radii of the bulges do not present any systematic trend with the filter.

The ratio of the scale length of the disk to the effective radius of the bulge, Fig. 14, presents a systematic decrease to the red. We also find a systematic increase

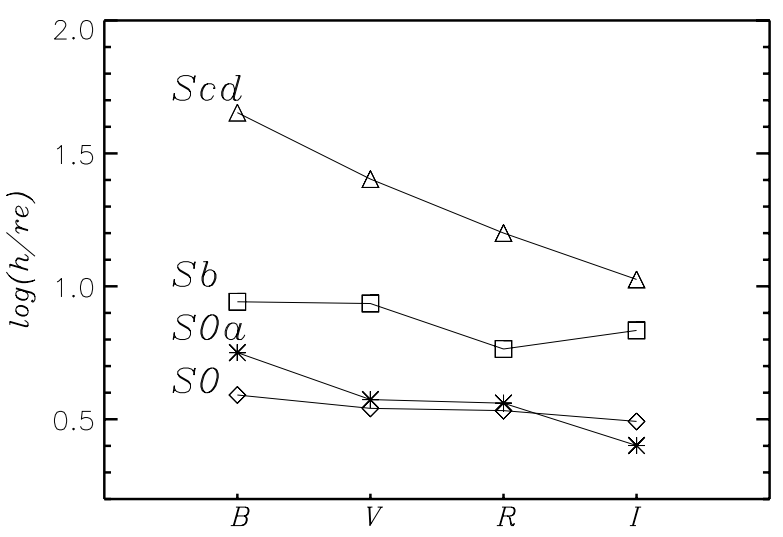

Fig. 14. The ratio $h / r_{\mathrm{e}}$ vs. the filter for those galaxies with well defined disk

of $r_{\mathrm{e}} / h$ from late to early type galaxies, contrary to that found by Courteau et al. (1996) and de Jong (1996). This problem has been rigorously treated in Graham \& Prieto (1999).

2.- Galaxies with an ill-defined disk.

Although in these galaxies the disk is not well defined, we have carried out the structural decomposition in order to determine which components are present and we have obtained an estimate of their scale lengths.

NGC 7013. This galaxy has a blue outer ring, seen clearly in the $B-V$ and $B-I$ images and in the luminosity profiles. This ring hinders the clear detection of the disk and this has been fitted to the external edges of the ring. The galaxy has an inclination close to the transparency limit and there is a trend toward decreasing $h$ in redder filters. We can conclude that this galaxy has a ring at $20^{\prime \prime}$, a lens of scale length is $50^{\prime \prime}$, and a bulge shape index of 1.5 .

NGC 7217. This galaxy has multiple blue and red rings which hinder clear detection of the disk. We have fitted the disk to the zone we consider to be between rings, 
but these rings are very wide and numerous except for the $I$ filter where they are smoother.

NGC 5992. This galaxy is probably interacting with NGC 5993. Except in the $B$ filter, there is too little data for the outer disk to make a good estimate of the disk parameters. We have fitted the disk to the outermost points of the galaxy, outside the bar region. We can conclude that this galaxy has a flat bar with scale length is $19^{\prime \prime}$.

NGC 7753. This galaxy has very strong spiral arms. We have carried out the fit of the disk in the inter-arm regions.

3.- Galaxies with large bars and well defined spiral arms.

These galaxies are, NGC 1300, NGC 7479, and NGC 7723. None of these galaxies have a well observed region of the disk due to the presence of powerful spiral arms. We have fitted the disk in individual profiles and on the existing points between the arms. There is no clear zone of disk in any of these galaxies. However, we have achieved the fittings to know the general features of the other components.

NGC 1300. This galaxy has a bar, a lens, prominent spiral arms and star formation regions.

NGC 7479 and NGC 7723. These galaxies have very few points to fit the disk. They have an elliptical bar with scale length of $51^{\prime \prime}$ and scale wide $25^{\prime \prime}$ in NGC 7479 and scale length of $22^{\prime \prime}$ and scale wide of $12^{\prime \prime}$ for NGC 7723 .

The bulges of the spiral galaxies in this sample, with the exception of NGC 1300, have an $n$ index which follows the general trend observed in spiral galaxies. The earliest, NGC 7217 (Sab), has an index $n=2.8$, and the rest have values between 1 and 2 .

We did not find Freeman type II profiles in this sample of 11 galaxies probably because we considered components other than the bulge and disk.

We found bars in half of the galaxies analyzed. Half of these bars have the same color as the underlying structure (NGC 1300, NGC 6056, and NGC 5992), and the other half are redder than such structure (NGC 7479, NGC 7723, and NGC 7753). The cause of this segregation is a question still to be understood. This could give us some indication of the state of the bar; the red bars are probably the oldest, and the young bars retain the color of the disk stars which give them their form.

\section{Summary and conclusions}

We have performed a structural decomposition for a sample of 11 disk galaxies of different morphological types. The bulges were fitted with an $r^{1 / n}$ law, the disks with an exponential law, the bars with elliptical or flat functions, rings and spiral arms with Gaussian functions, and the lenses with a quadratic expression. Prior to the fit, we used the $U, B, V, R$, and $I$ photometric data (color and colorindex images and luminosity, ellipticity, and position-angle profiles) to decide the type and number of different components which form the galaxies and to estimate their scale lengths. We have written an interactive profile-fitting routine for the decomposition, which fits the parameters of the models in an iterative process. We find and model all components which form the galaxies: bulge, disk, bar, lens, ring, etc. Only for galaxies with well defined disks do we give reliable parameters for the bulge and disk.

For the galaxies with well defined disks we find that:

- The scale length of the disk decreases when the filter is redder, as would be expected for an optical thickness increasing towards the center of the galaxy;

- There is a increased trend in the index $n$ of the bulge with the redder filters. This can be interpreted as the older population being more extended than the younger;

- The ratio between the scale length of the disk and the effective radius of the bulge, shows a systematic decrease to the red. We also find a systematic increase in this ratio from late to early types of galaxies.

For the galaxies with ill-defined disk we have given the position and length-scales of components other than the bulge and disk.

We do not find Freeman type II profiles in this sample of galaxies, probably because we considered components other than the bulge and disk.

We found bars in half of the galaxies analyzed, which are either elliptical or flat. Half of these bars have the same color as the underlying structure (NGC 1300, NGC 6056, and NGC 5992), and the other half have redder colors (NGC 7479, NGC 7723, and NGC 7753).

Acknowledgements. We are most sincerely grateful to Alister Graham for useful conversations and kindly reading and improving the manuscript. We express our thanks to Terry Mahoney for correcting the English of the manuscript. The 2.5-m INT is operated on the island of La Palma by the Royal Greenwich Observatory at the Spanish Observatorio del Roque de Los Muchachos of the Instituto de Astrofísica de Canarias. Support for this work comes from project PB97-1107 and PB97-0219 of the Spanish DGES. The observations received financial support from the European Commission through the Access to Large-Scale Facilities Activity of the Human Capital and Mobility Programme.

\section{References}

Aguerri, J. A. L., Varela, A. M., Prieto, M., \& Muñoz-Tuñón, C. 1999, AJ, 119, 1638

Andredakis, Y. C., \& Sanders, R. M. 1994, MNRAS, 267, 283

Athanassoula, E. 1992a, MNRAS, 259, 328

Athanassoula, E. 1992b, MNRAS, 259, 345

Balzano, V. A. 1983, ApJ, 268, 602

Bicay, M. D., Kojoian, G., Seal, J., Dickinson, D. F., \& Malkan, H. A. 1995, ApJS, 98, 369

Boroson, T. 1981, ApJS, 46, 177

Buta, R., van Driel, W., Braine, J., et al. 1995, ApJ, 450, 593

Capaccioli, M., Held, E. V., \& Nieto, J.-L. 1987, AJ, 94, 1519

Courteau, S., de Jong, R. S., \& Broeils, A. H. 1996, ApJ, 457, L73

Devereux, N. A. 1989, ApJ, 346, 126 
Duval, M. F., \& Athanassoula, E. 1983, A\&A, 121, 297

Elmegreen, B. G., \& Elmegreen, D. M. 1985, ApJ, 288, 438

Elmegreen, B. G., Elmegreen, D. M., Chromey, F. R., Hasselbacher, D. A., \& Bisseli, B. A. 1996, AJ, 111, 2233

Engelbracht, C. W., Rieke, M. J., Rieke, G. M., \& Latter, W. B. 1996, ApJ, 467, 227

Eskridge, P. B., \& Pogge, R. W. 1991, AJ, 101, 2056

Freeman, K. C. 1966, MNRAS, 133, 47

Giurcin, G., Tamburini, L., Mardirossian, F., Mezzetti, H., \& Monaco, P. 1994, ApJ, 427, 202

Graham, A., \& Prieto, M. 1999, ApJ, 524, 23L

Ho, L. C., Filippenko, A. V., \& Sargent, W. L. W. 1993, ApJ, 417, 63

Hummel, E., van der Hulst, J. M., Keel, W. C., \& Kennicutt, R. C. 1987, A\&AS, 70, 517

Hua, C. T., Donas, J., \& Duun, H. H. 1980, A\&A, 90, 8

de Jong, R. S. 1996, A\&AS, 118, 557

Keel, W. C. 1983a, ApJ, 269, 466

Keel, W. C. 1983b, ApJ, 268, 632

Keel, W. C. 1984, ApJ, 282, 75

Kent, S. M. 1987, AJ, 93, 816

Knapp, G. R., van Driel, W., Schwarz, V. J., van Woerden, H., \& Gallager, J. S. 1984, A\&A, 133, 127

Kormendy, J. 1977, ApJ, 214, 359

Laine, S. J. 1996, Ph.D. Thesis, Florida University

Lynds, B. T. 1974, ApJS, 28, 391

Mazzarella, J. M., \& Balzano, V. A. 1986, ApJS, 62, 751
Moriondo, G., Giovanardi, C., \& Hunt, L. K. 1997, A\&AS, 130, 81

Prieto, M., Beckman, J. E., Cepa, J., \& Varela, A. M. 1992b, A\&A, 257, 85

Prieto, M., Gottesman, S. T., Aguerri, J. A. L., \& Varela, A. M. 1997, AJ, 114, 1413

Prieto, M., Longley, D. P. T., Pérez, E., et al. 1992a, A\&AS, 93, 557

Salo, H., \& Laurikainen, E. 1993, ApJ, 410, 586

Schombert, J. M., \& Bothun, G. D. 1987, AJ, 92, 1, 60

Sersic, J. L. 1968, Atlas de galaxias australes Córdoba: Observatorio Astronónico

Sersic, J. L. 1982, Extragalactic Astronomy (Reidel, Dordrecht)

Spark, W. B. 1988, AJ, 1569, NASA ADS

de Vaucouleurs, G., de Vaucouleurs, A., Corwin, H. G., \& Buta, R. 1991, Third Reference Catalog of Bright Galaxies (New York: Springer) (RC3)

de Vaucouleurs, G. 1958, Ann. Astrophys., 11, 247

Varela, A. M., Muñoz-Tuñón, C., \& Simmoneau, E. 1996, A\&A, 306, 381

Verdes-Montengro, L., Bosma, A., \& Athanassoula, E. 1995, A\&A, 300, 65

Wozniak, H., Friedli, D., Martinet, L., Martin, P., \& Bratschi, P. 1995, A\&AS, 111, 115

Xilouris, E. M., Byun, Y. I., Kylafis, N. D., Paleologou, E. U., \& Papamastorakis, J. 1999, A\&A, 344, 868 